\title{
Examining Ionic Liquid Effects on Mononuclear Rearrangement of Heterocycles
}

using QM/MM Simulations

Caley Allen, Robel Ghebreab, Brian Doherty, Bin Li, and Orlando Acevedo*

Department of Chemistry, University of Miami, Coral Gables, Florida 33146

E-mail: orlando.acevedo@miami.edu

Supporting Material 


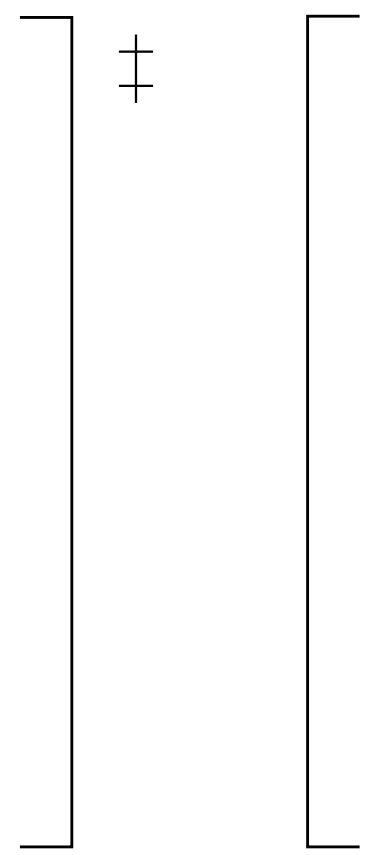

Specific Base Catalyzed

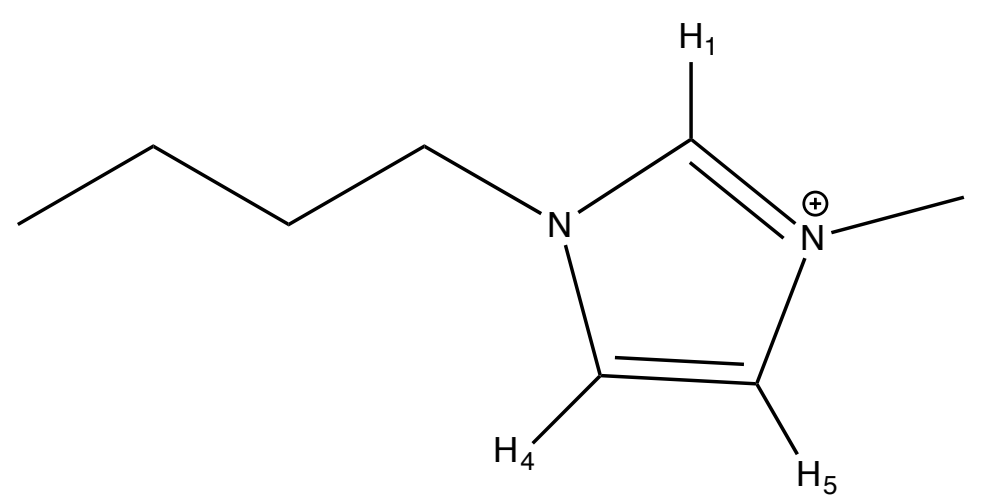

[BMIM]
$\left[\mathrm{BF}_{4}\right]$

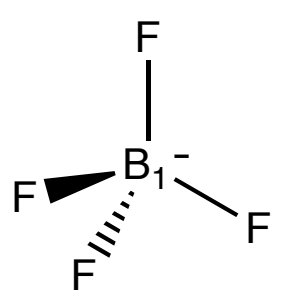

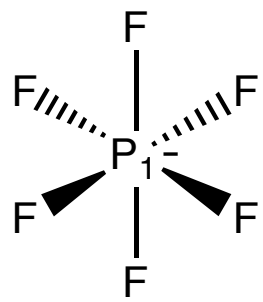

$\left[\mathrm{PF}_{6}\right]$

Figure S1. Structures of the ions and substrate with atom and functional group labels used for the corresponding figures. 


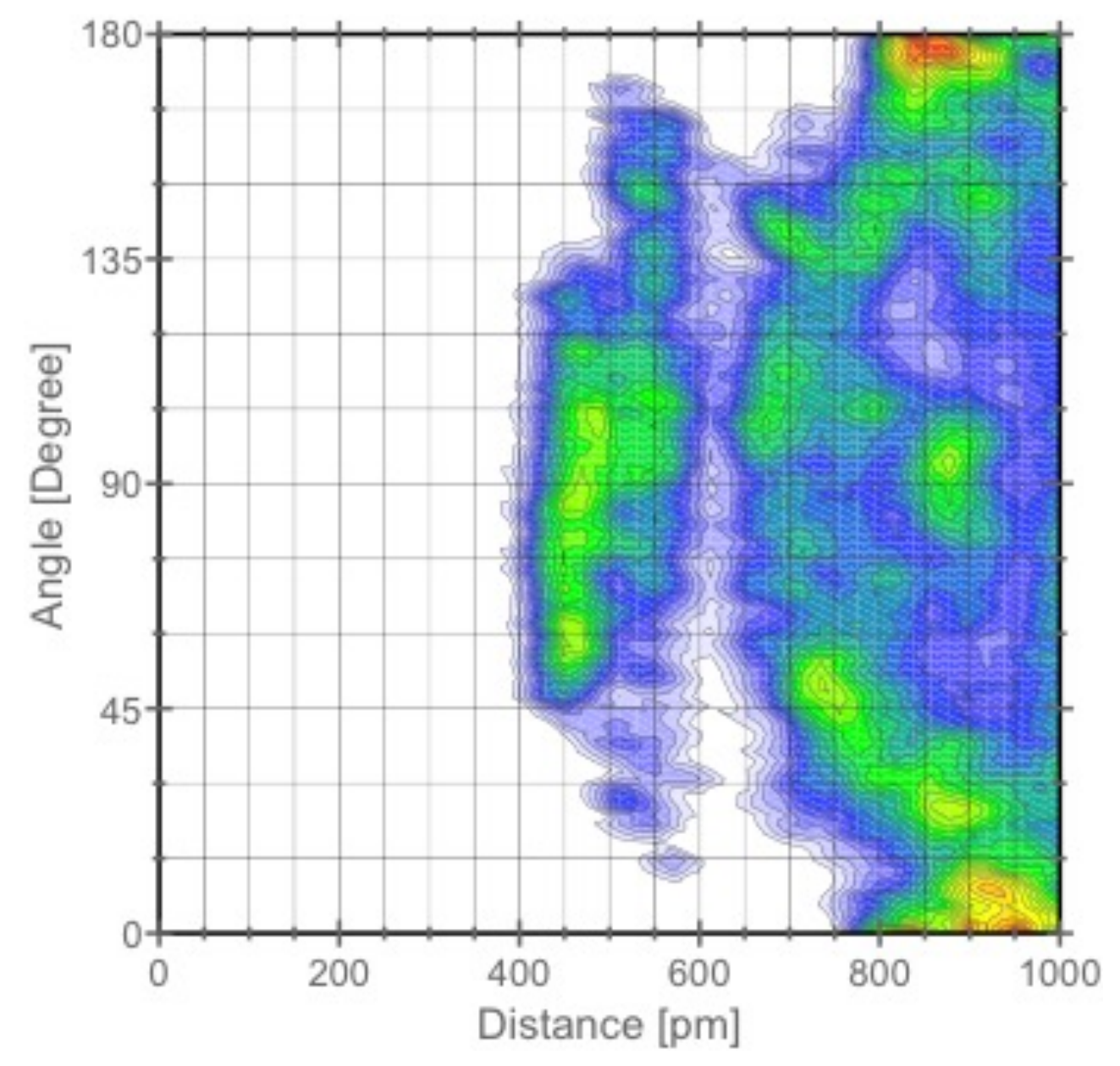

GS-Catalyzed

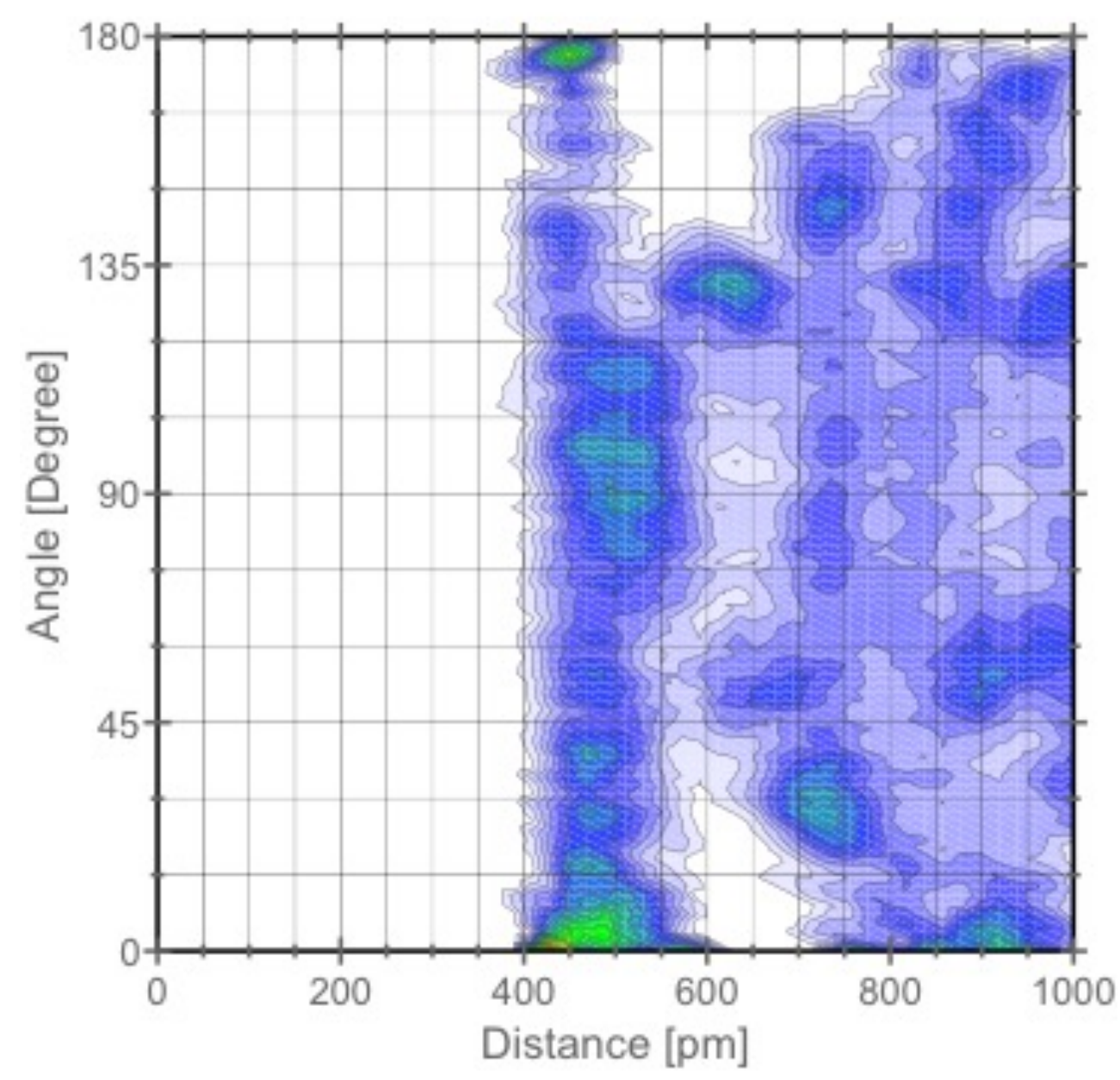

GS-Uncatalyzed

Figure S2. Combined distribution functions assessing the $\pi^{+}-\pi$ stacking between the imidazolium cation and Ring 1 (R1) of

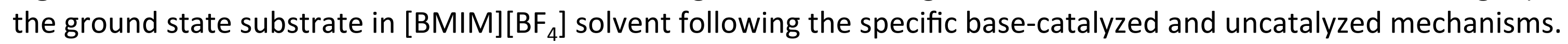




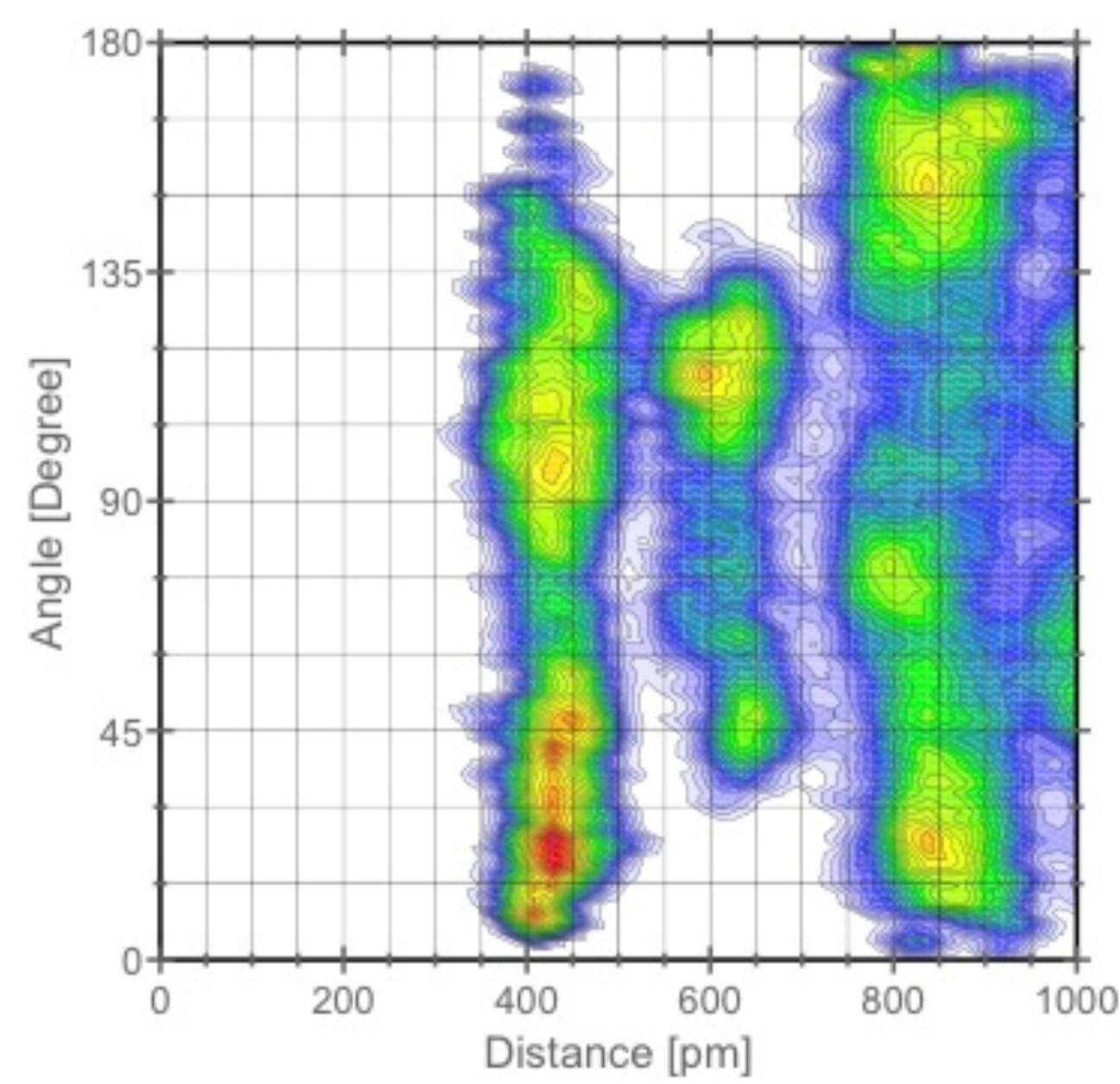

GS-Catalyzed

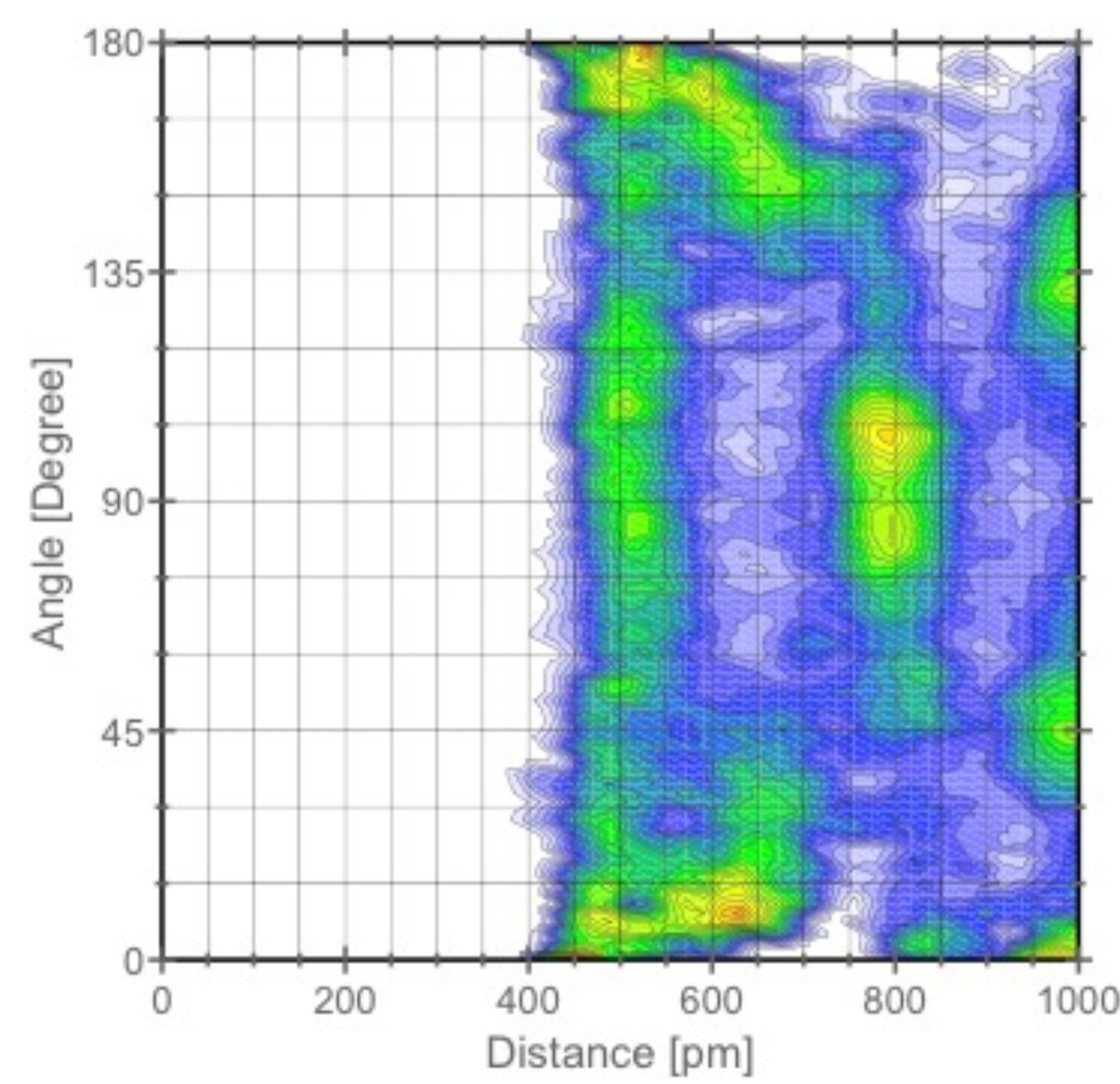

GS-Uncatalyzed

Figure S3. Combined distribution functions assessing the $\pi^{+}-\pi$ stacking between the imidazolium cation and Ring 2 (R2) of

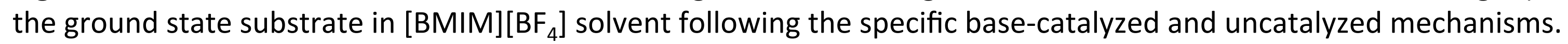




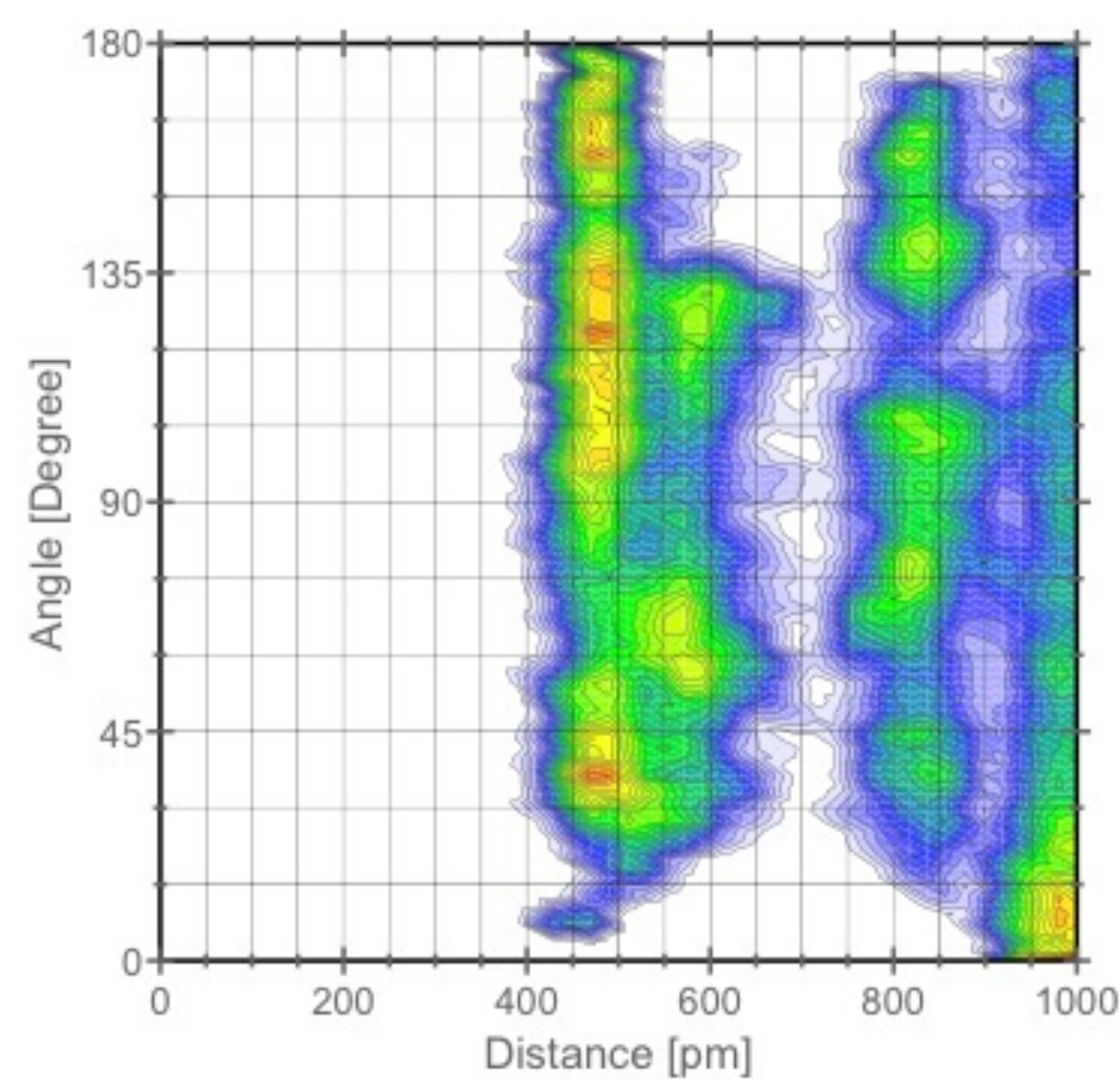

GS-Catalyzed

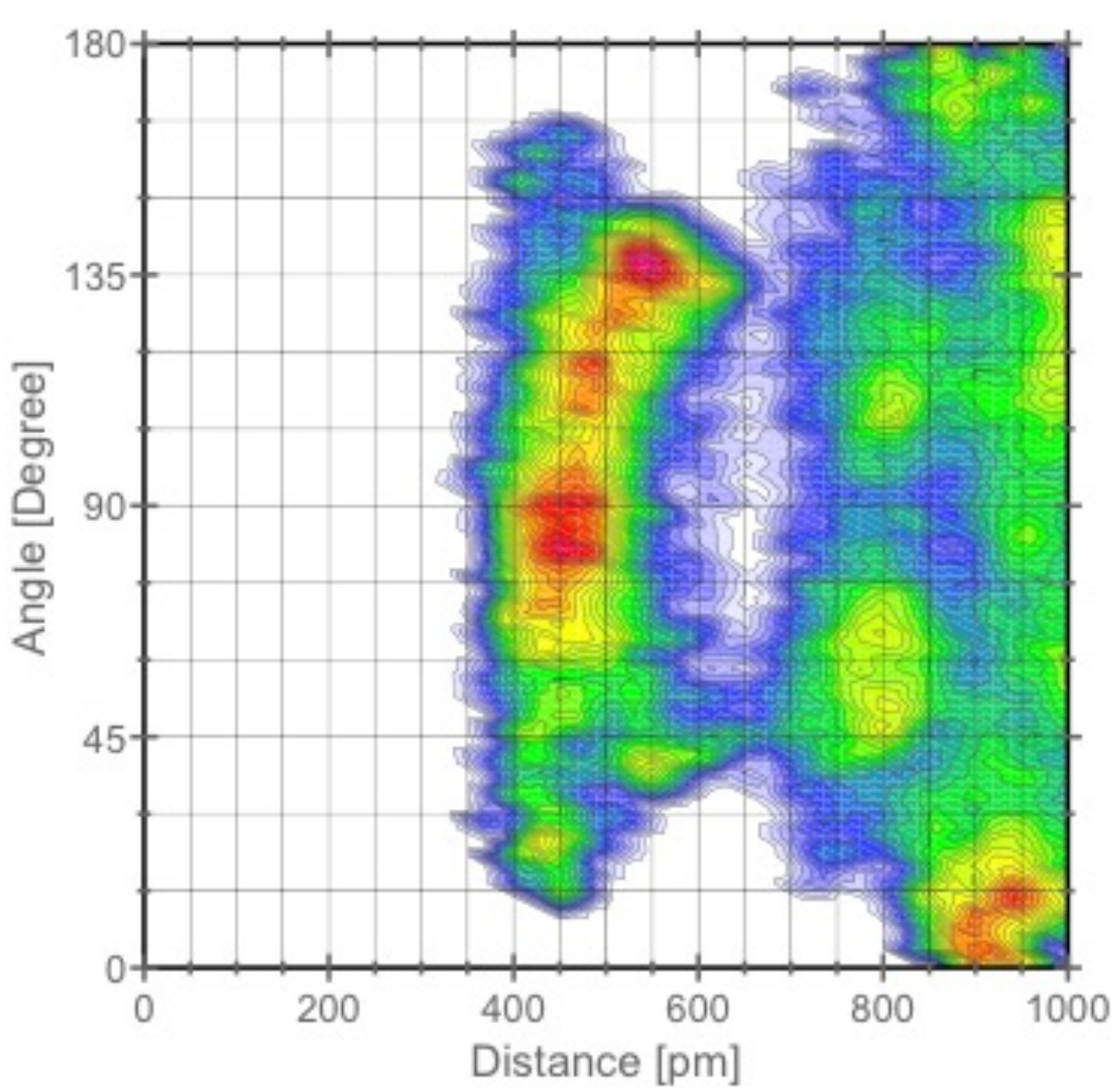

GS-Uncatalyzed

Figure S4. Combined distribution functions assessing the $\pi^{+}-\pi$ stacking between the imidazolium cation and Ring 3 (R3) of the ground state substrate in $[\mathrm{BMIM}]\left[\mathrm{BF}_{4}\right]$ solvent following the specific base-catalyzed and uncatalyzed mechanisms. 


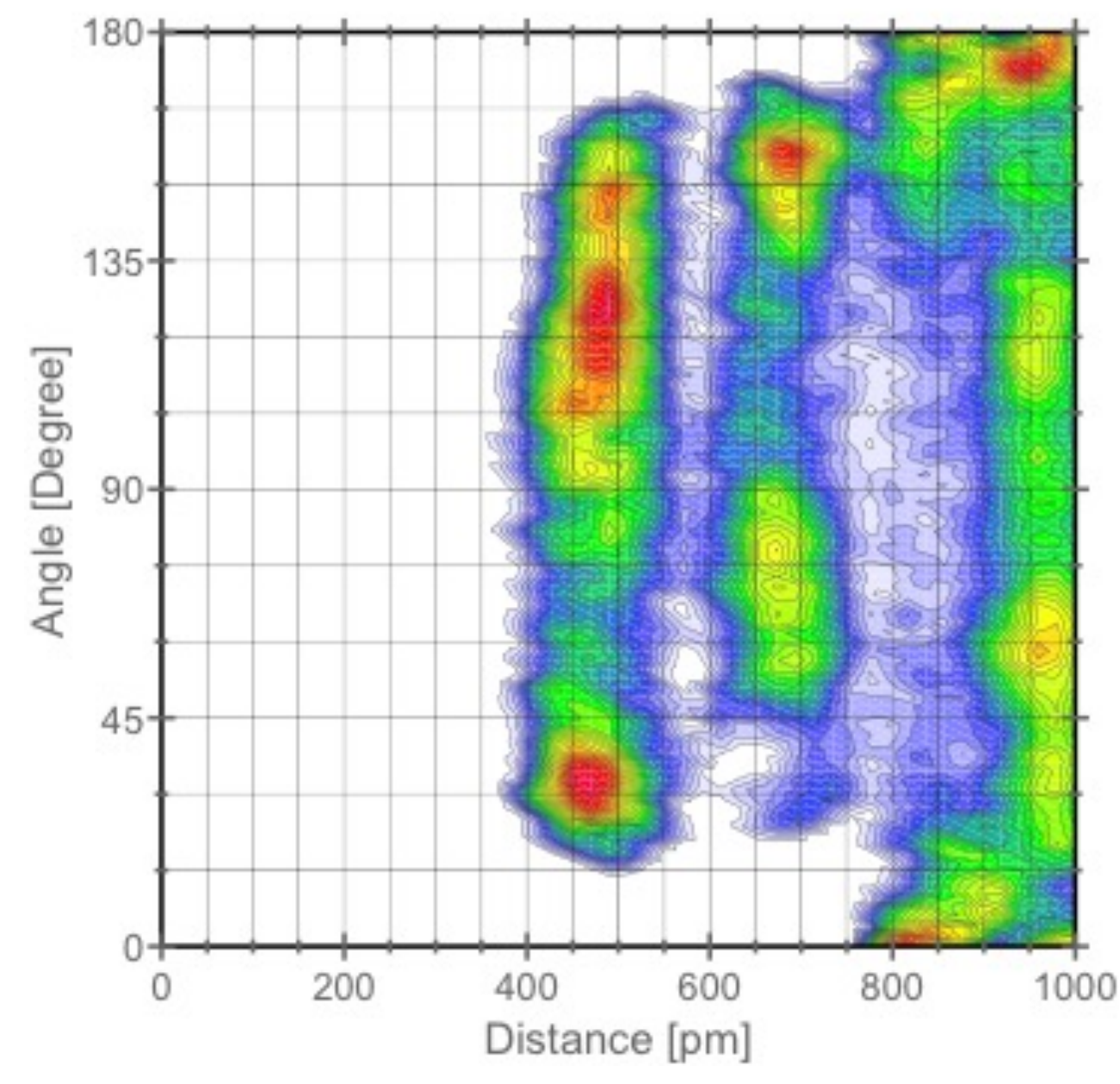

GS-Catalyzed

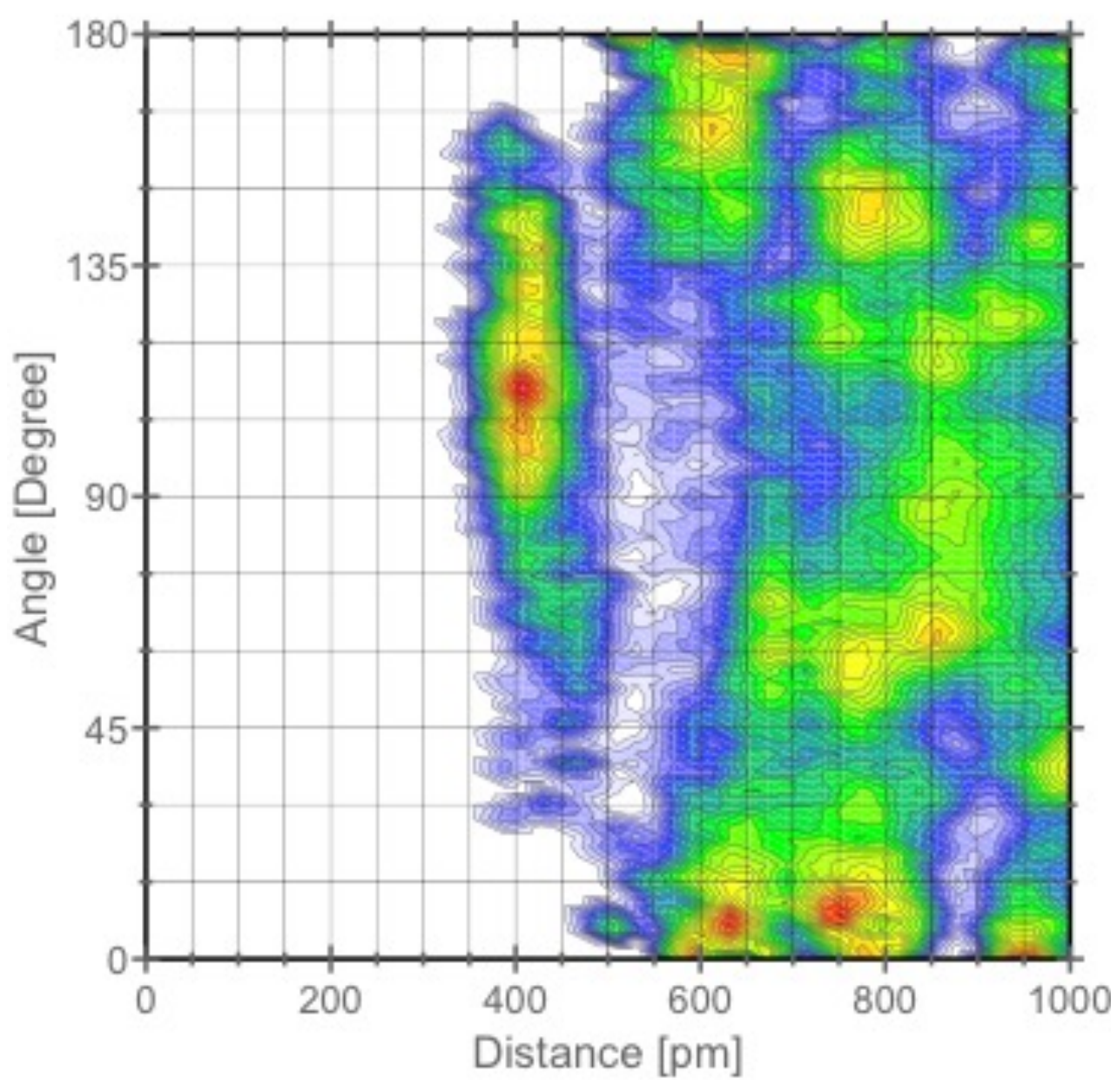

GS-Uncatalyzed

Figure S5. Combined distribution functions assessing the $\pi^{+}-\pi$ stacking between the imidazolium cation and Ring 1 (R1) of the ground state substrate in $[B M I M]\left[\mathrm{PF}_{6}\right]$ solvent following the specific base-catalyzed and uncatalyzed mechanisms. 


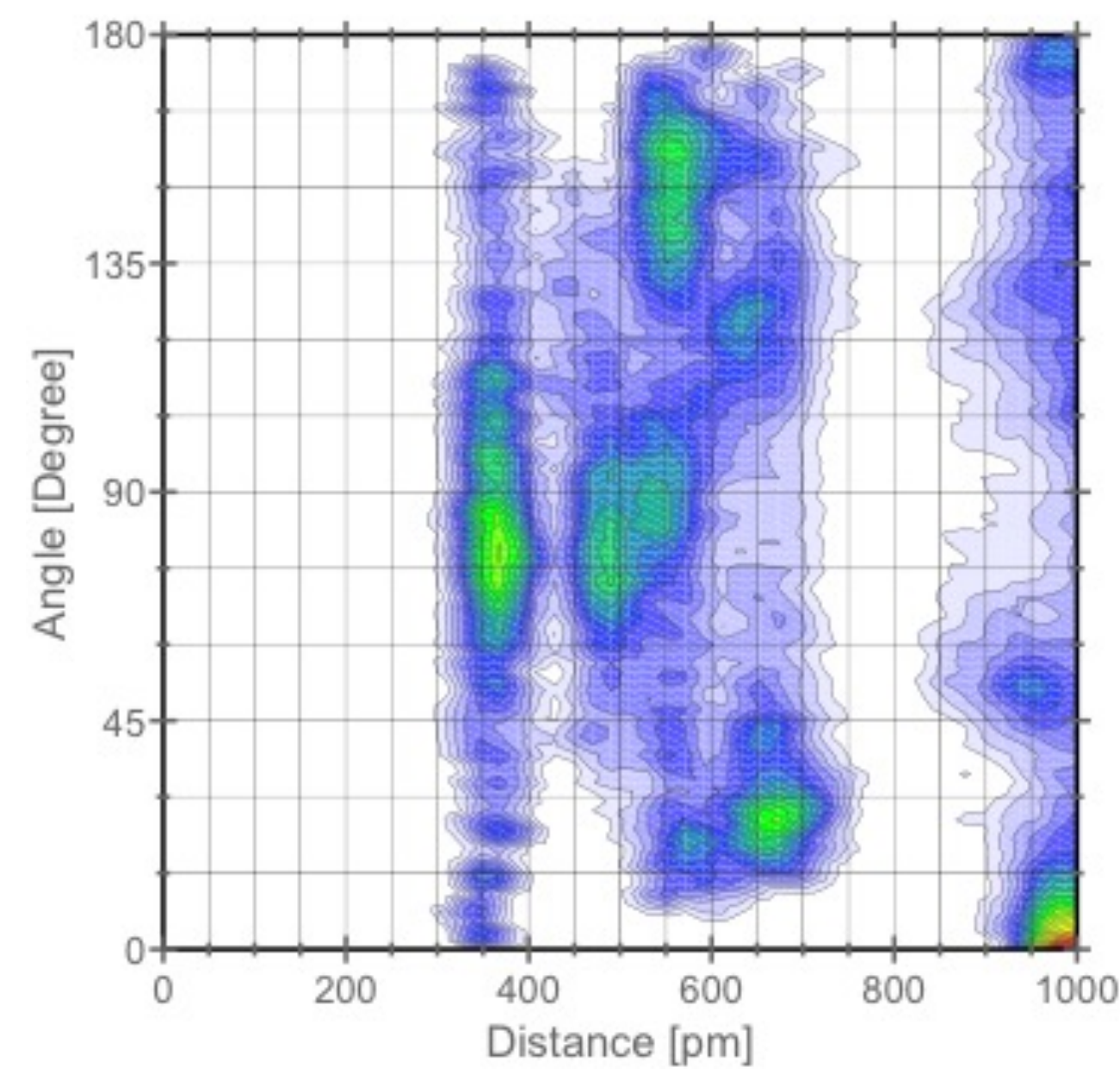

GS-Catalyzed

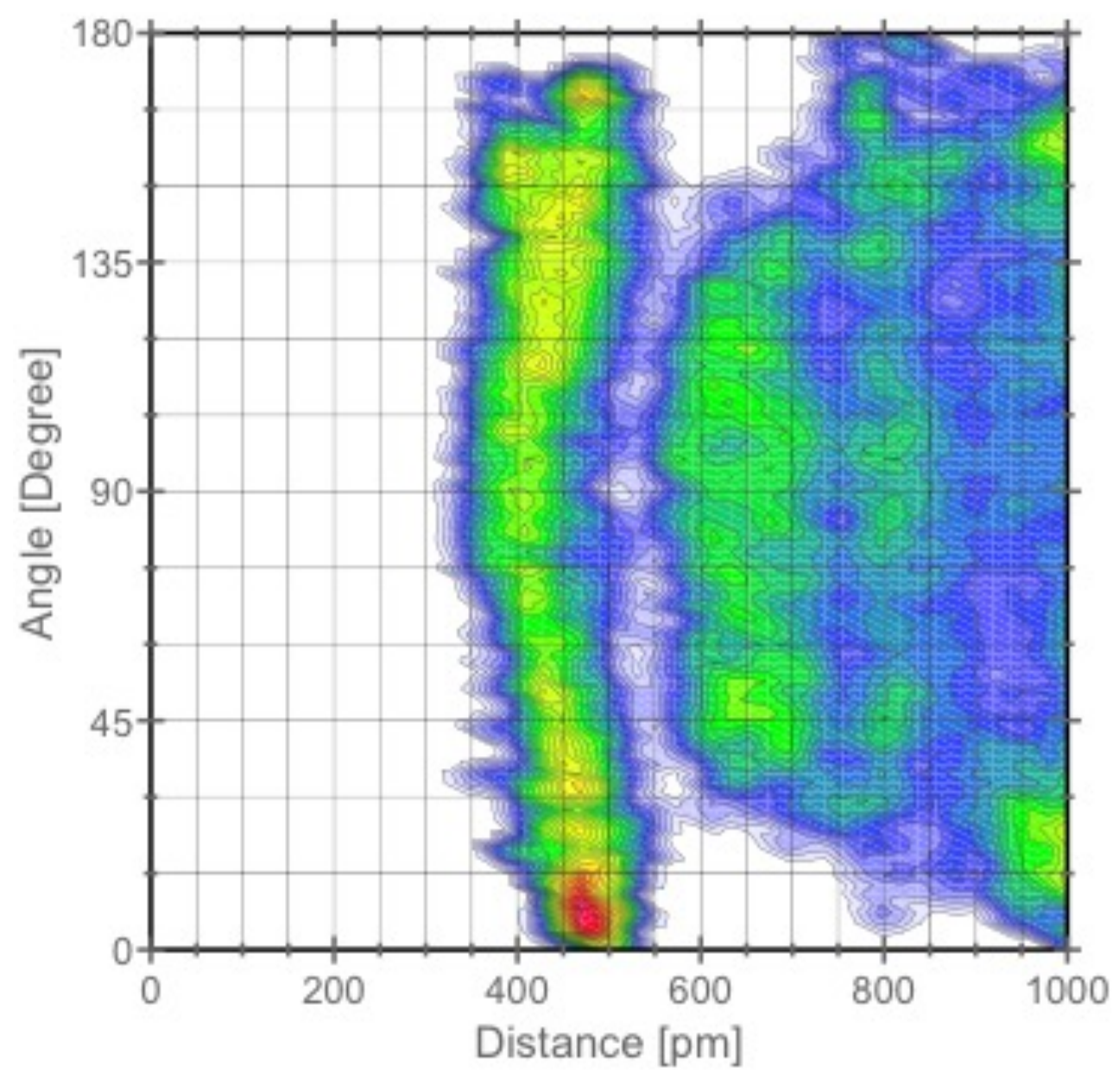

GS-Uncatalyzed

Figure S6. Combined distribution functions assessing the $\pi^{+}-\pi$ stacking between the imidazolium cation and Ring 2 (R2) of the ground state substrate in $[B M I M]\left[\mathrm{PF}_{6}\right]$ solvent following the specific base-catalyzed and uncatalyzed mechanisms. 


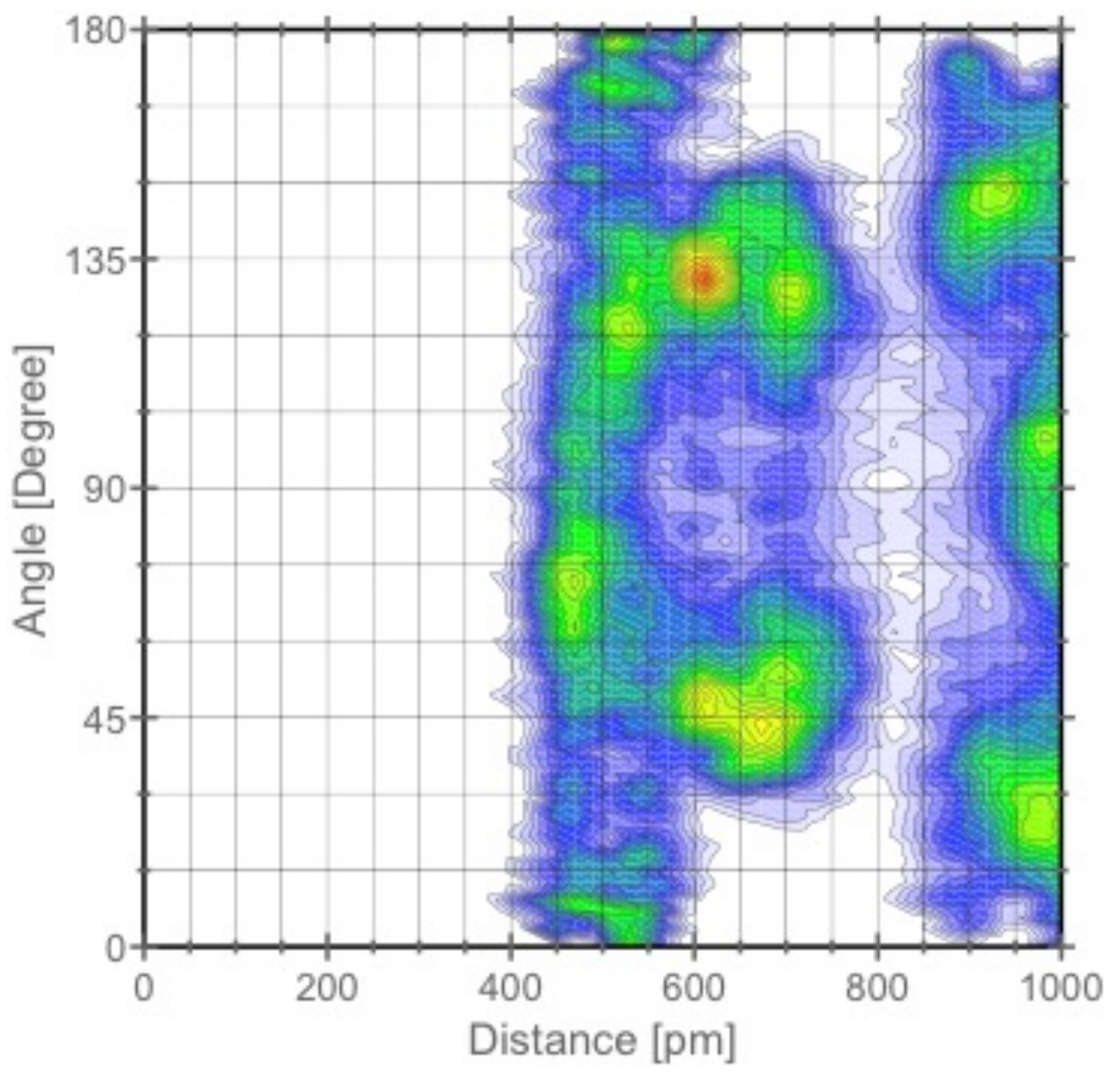

GS-Catalyzed

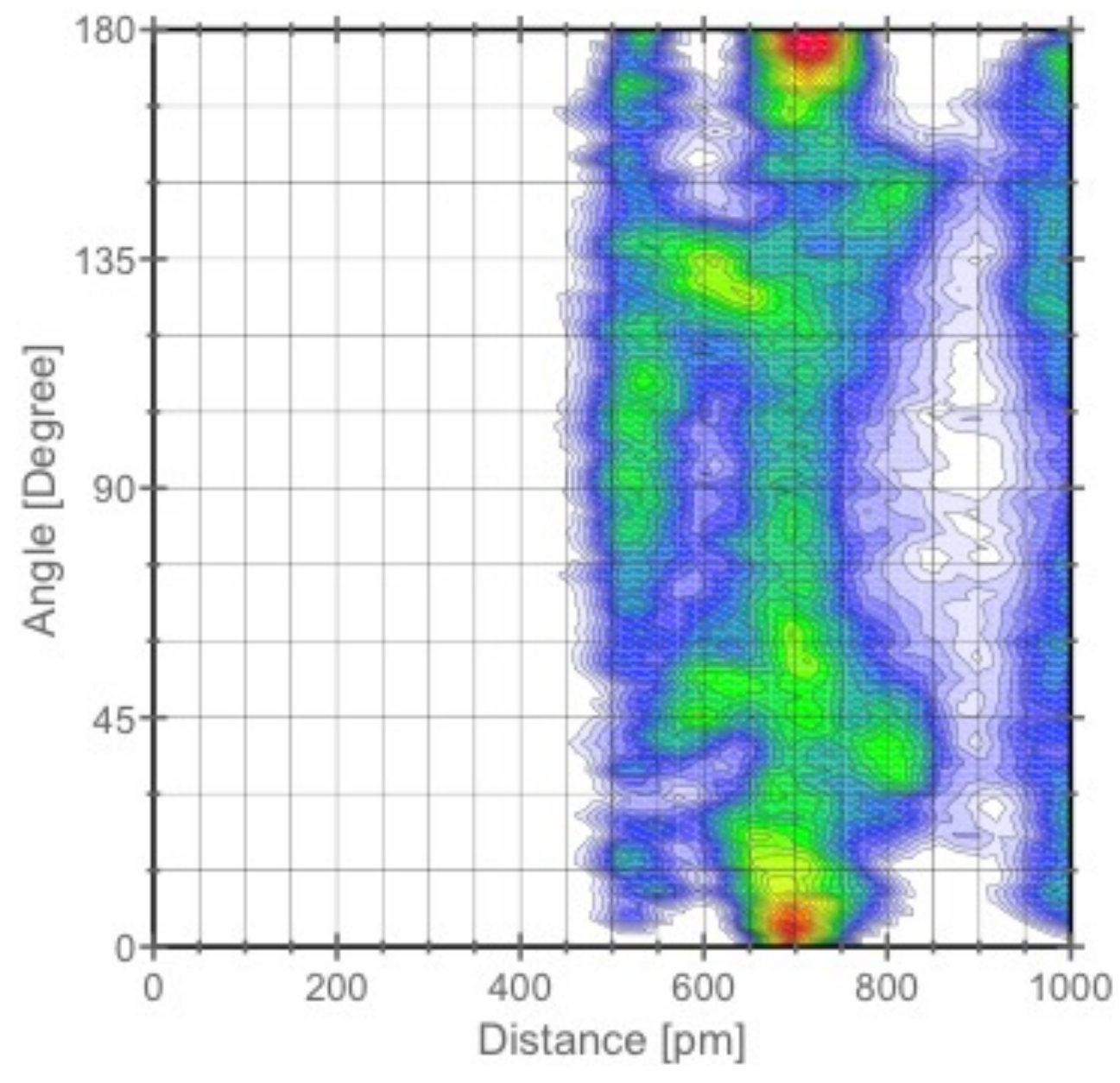

GS-Uncatalyzed

Figure S7. Combined distribution functions assessing the $\pi^{+}-\pi$ stacking between the imidazolium cation and Ring 3 (R3) of the ground state substrate in $[B M I M]\left[\mathrm{PF}_{6}\right]$ solvent following the specific base-catalyzed and uncatalyzed mechanisms. 


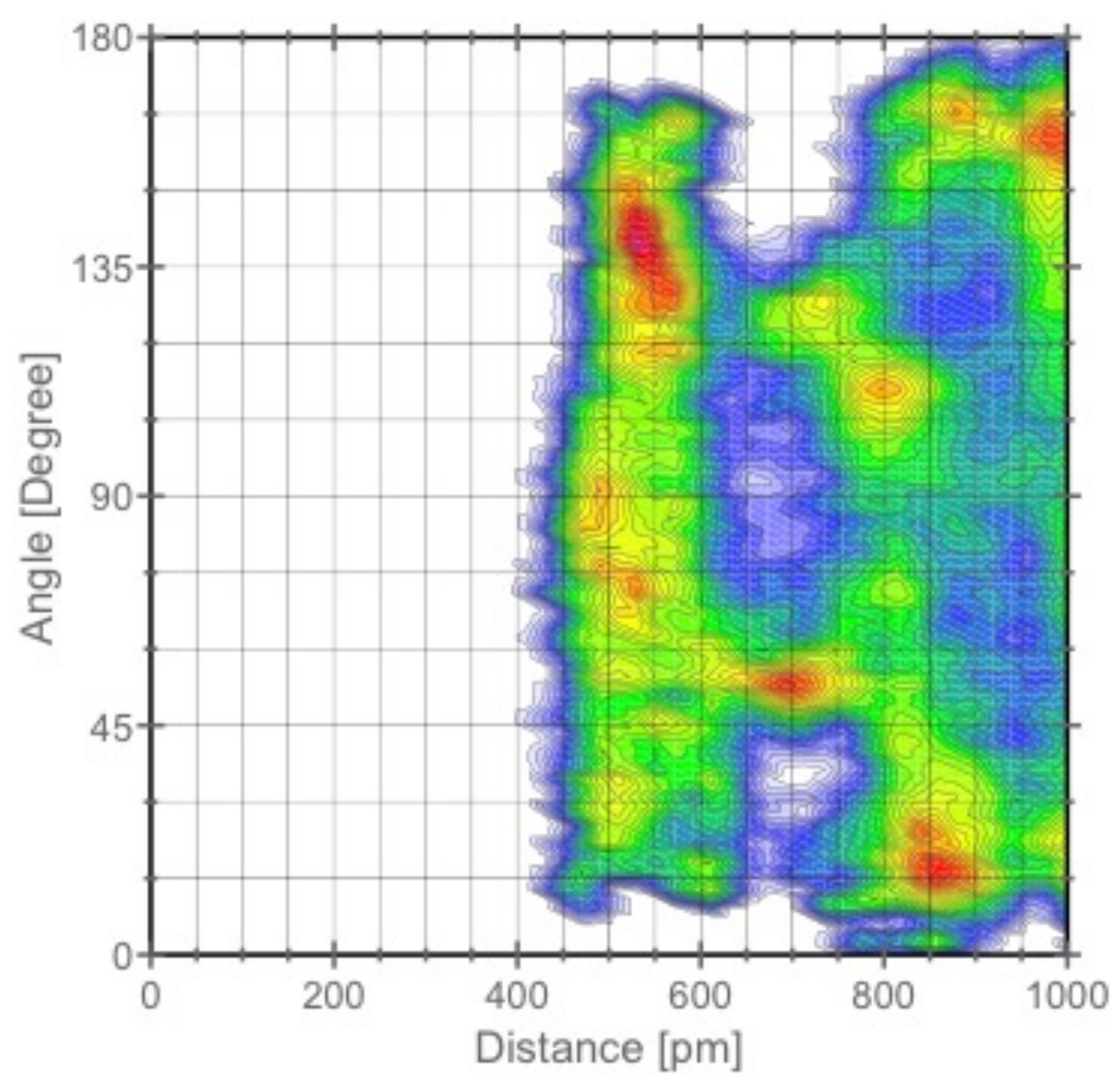

TS-Catalyzed

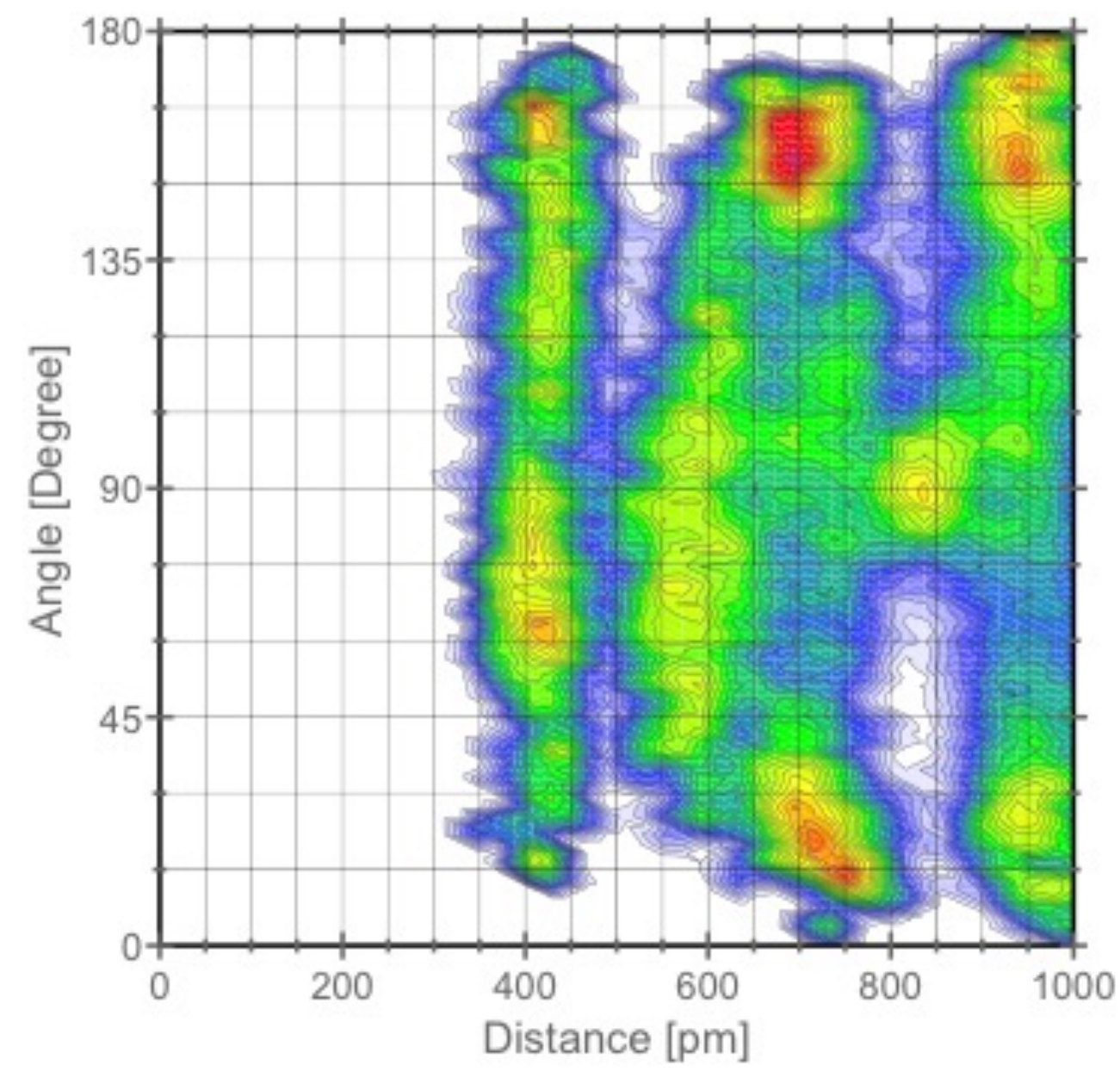

TS-Uncatalyzed

Figure S11. Combined distribution functions assessing the $\pi^{+}-\pi$ stacking between the imidazolium cation and Ring 1 (R1) of

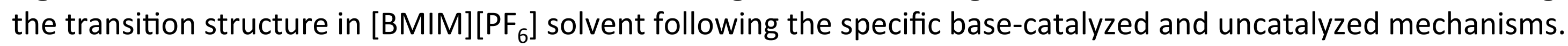




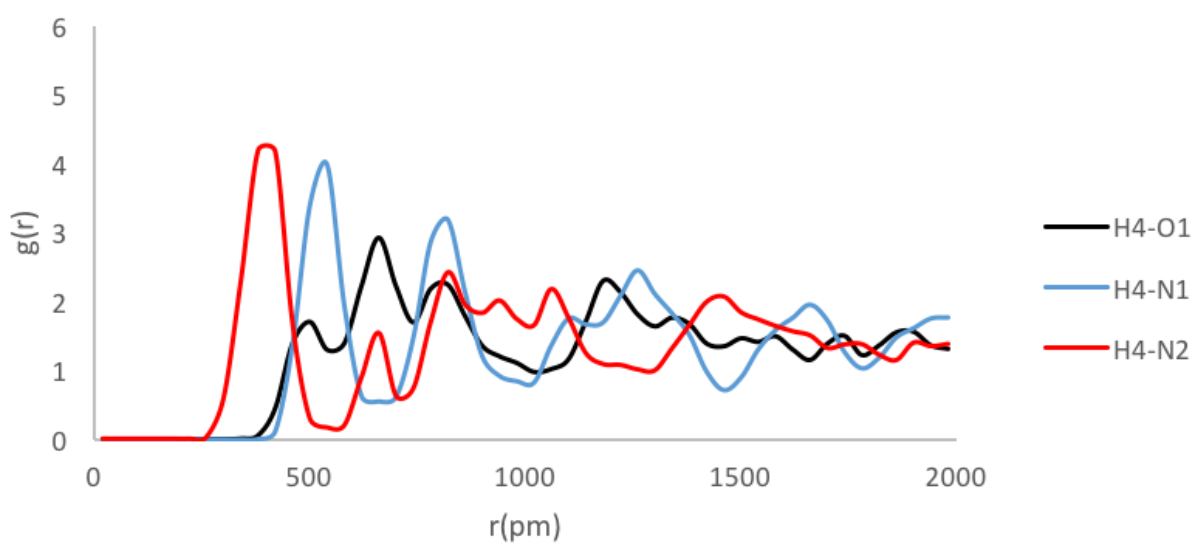

Specific Base Catalyzed [BMIM] $\left[\mathrm{BF}_{4}\right]$ Transition State

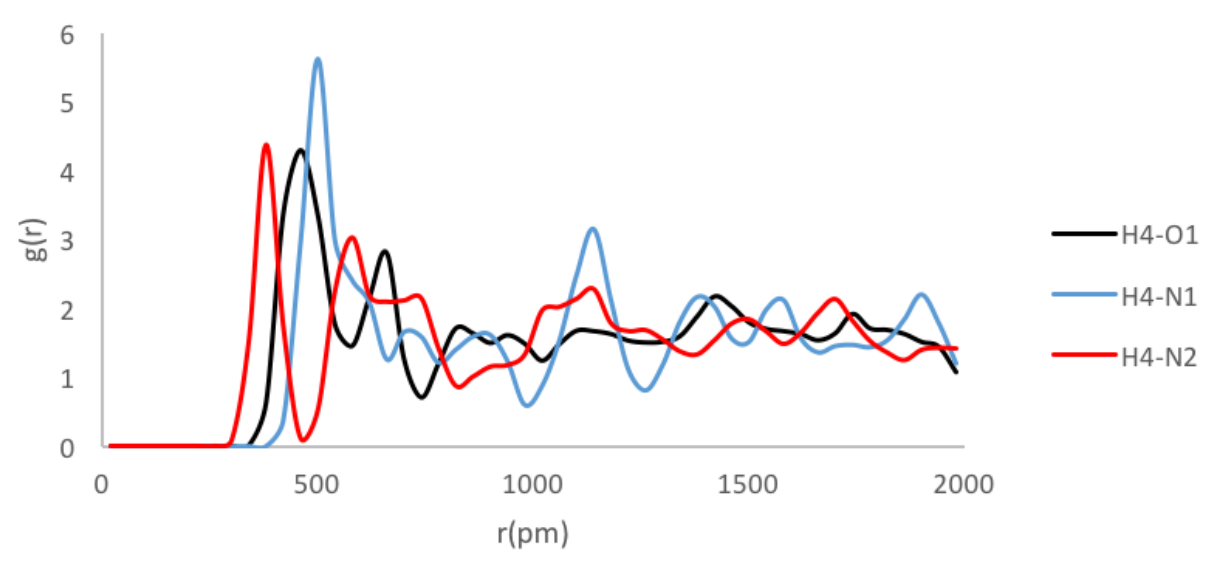

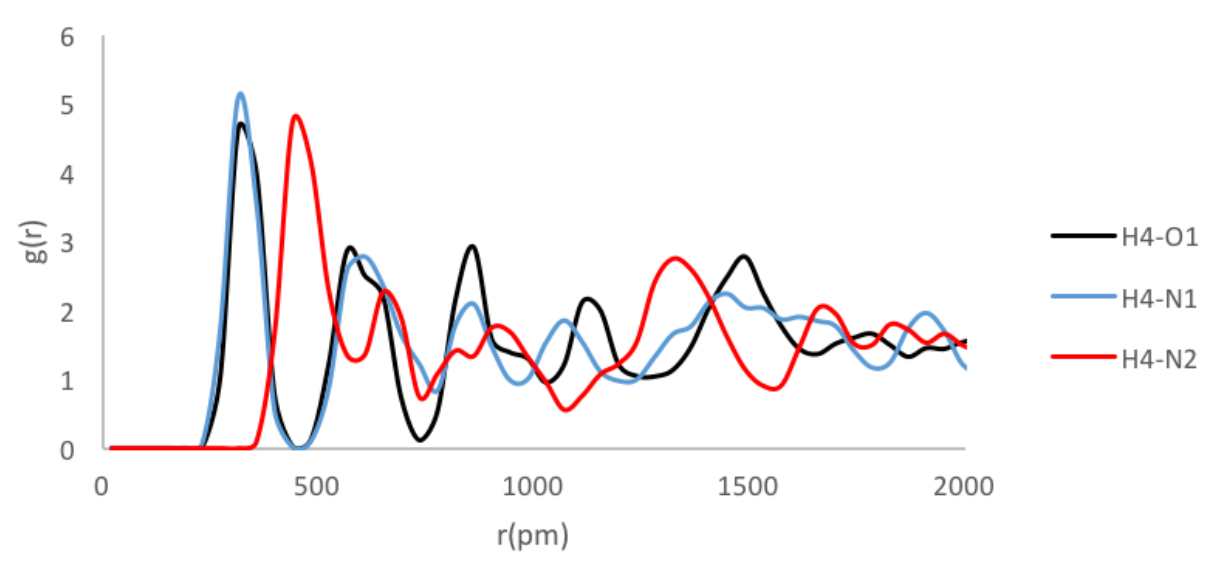

Specific Base Catalyzed [BMIM] $\left[\mathrm{PF}_{6}\right]$ Transition State

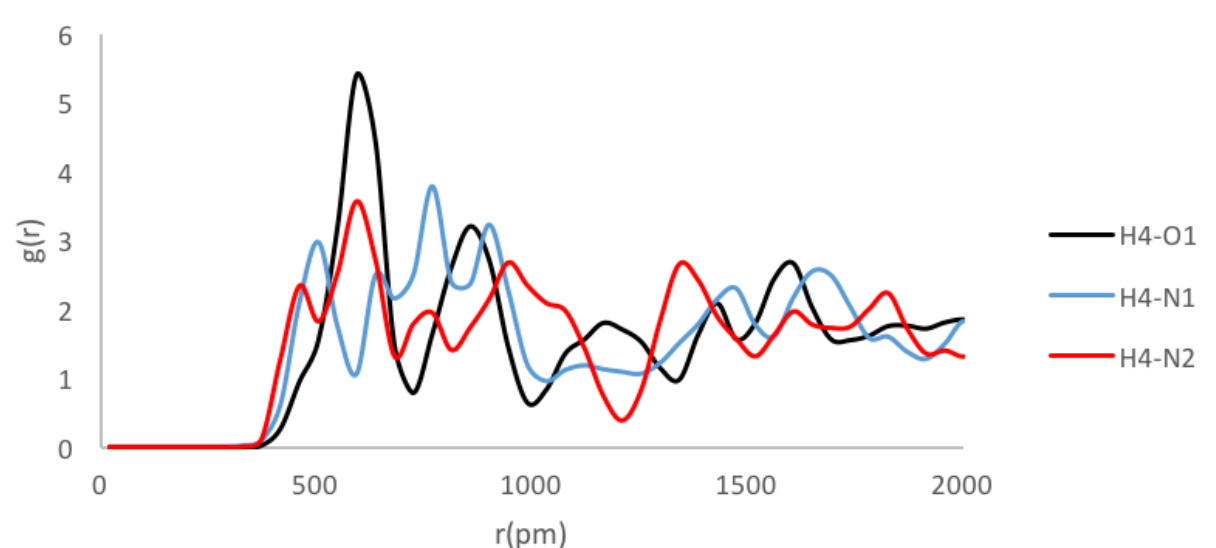

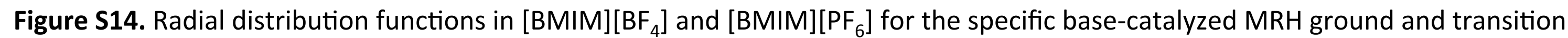
states between the $\mathrm{H} 4$ proton on BMIM and the oxadiozolic oxygen (H4-O1, black), oxadiozolic nitrogen (H4-N1, blue), and hydrazonic nitrogen (H4-N2, red). 

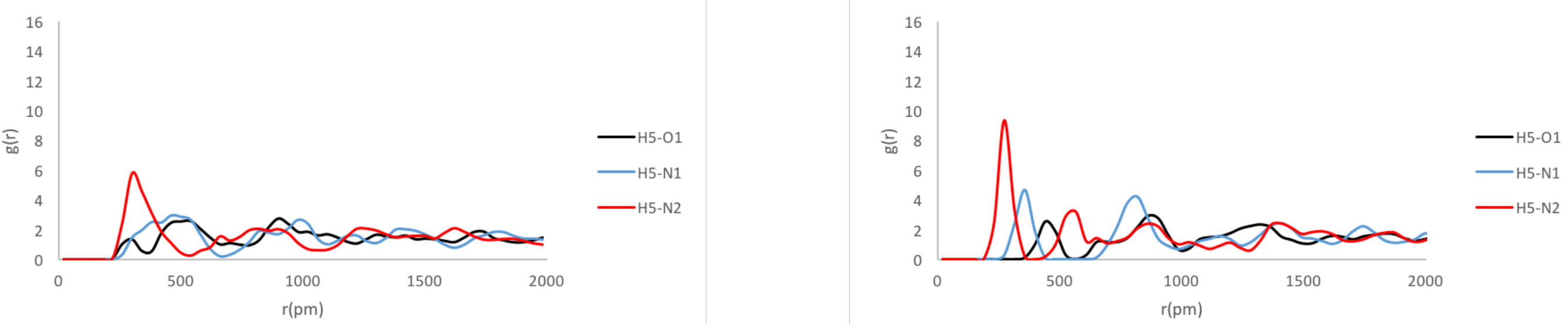

Specific Base Catalyzed [BMIM] $\left[\mathrm{BF}_{4}\right]$ Transition State

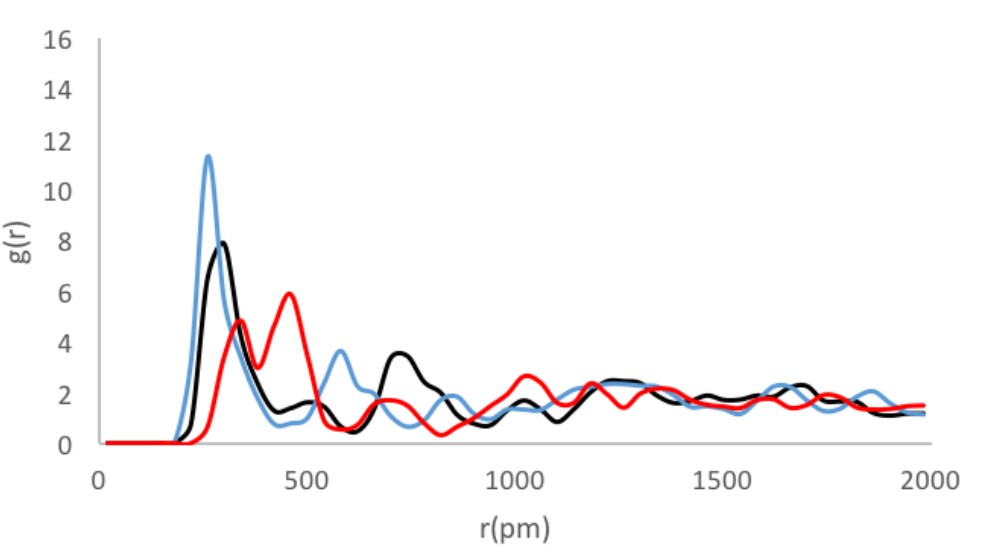

Specific Base Catalyzed [BMIM][PF$\left.F_{6}\right]$ Transition State

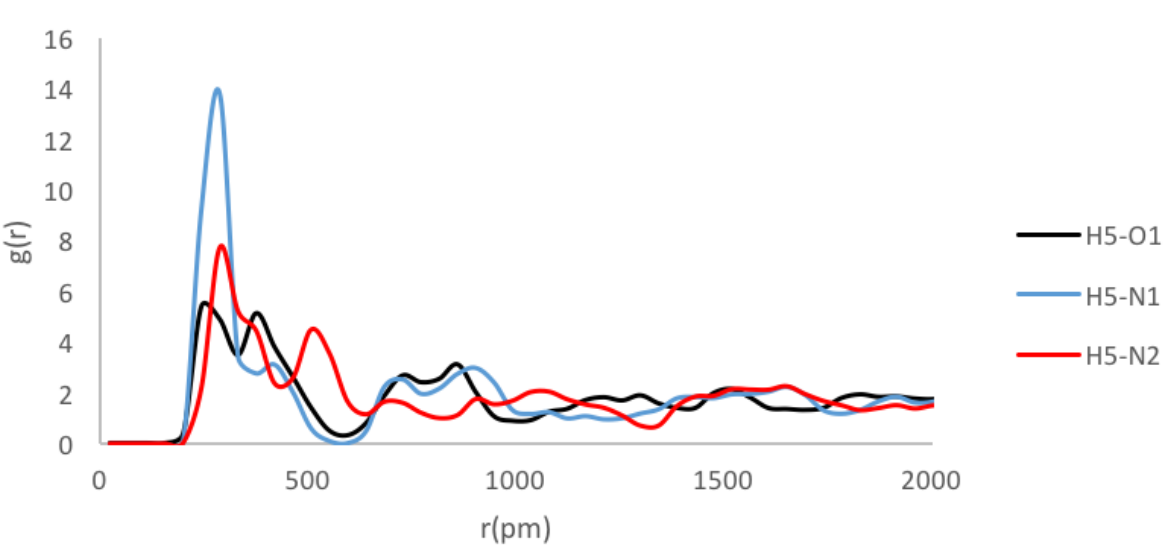

Figure S15. Radial distribution functions in $\left[\mathrm{BMIM}^{-}\left[\mathrm{BF}_{4}\right]\right.$ and $[\mathrm{BMIM}]\left[\mathrm{PF}_{6}\right]$ for the specific base-catalyzed MRH ground and transition states between the $\mathrm{H} 5$ proton on BMIM and the oxadiozolic oxygen (H5-O1, black), oxadiozolic nitrogen (H5-N1, blue), and hydrazonic nitrogen (H5-N2, red). 

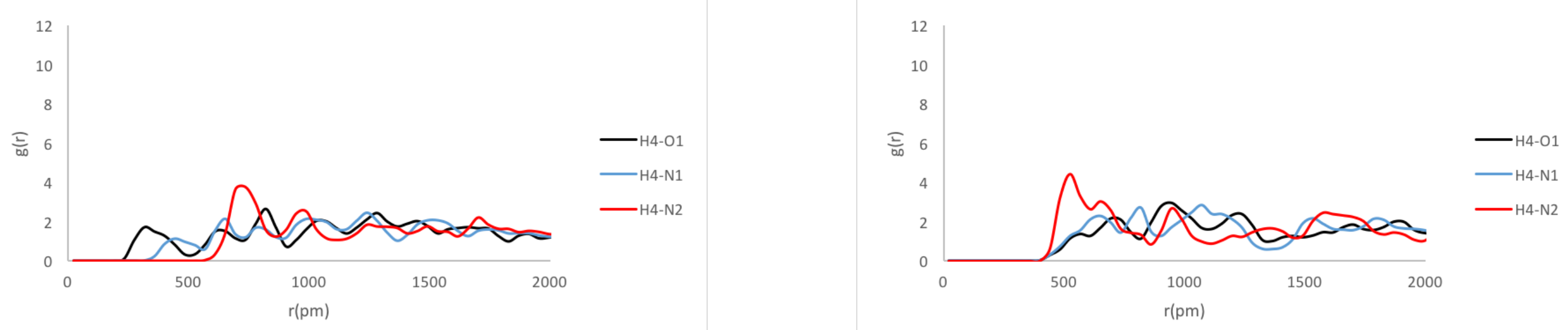

Uncatalyzed $[\mathrm{BMIM}]\left[\mathrm{BF}_{4}\right]$ Transition State

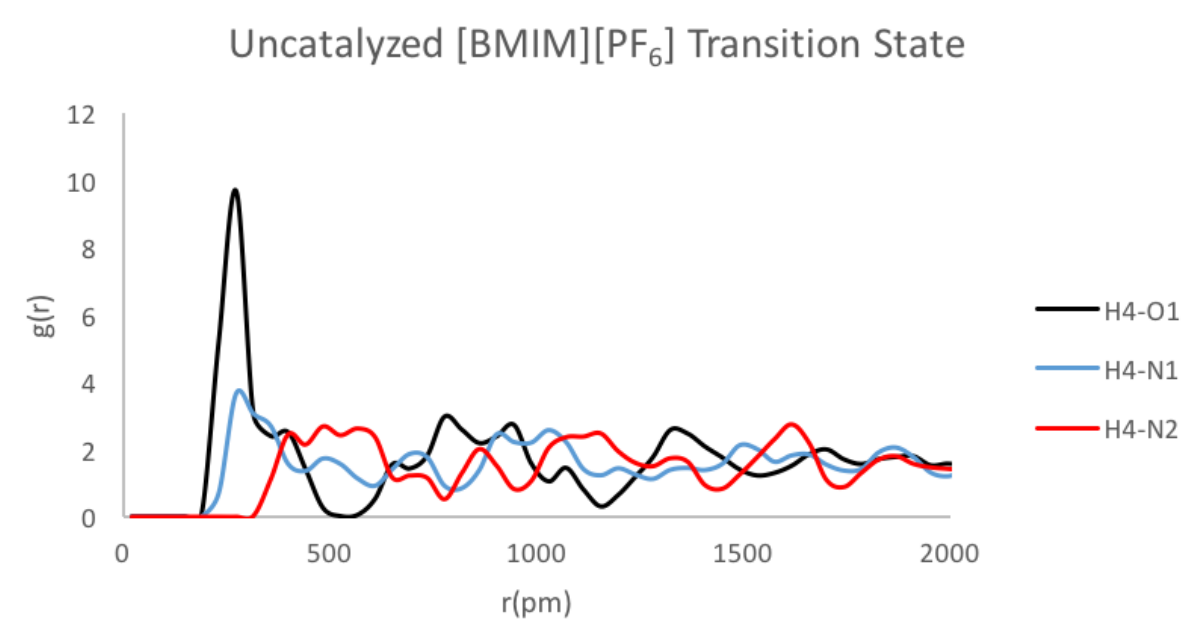

Figure S16. Radial distribution functions in $[\mathrm{BMIM}]\left[\mathrm{BF}_{4}\right]$ and $[\mathrm{BMIM}]\left[\mathrm{PF}_{6}\right]$ for the uncatalyzed $\mathrm{MRH}$ ground and transition states between the $\mathrm{H} 4$ proton on BMIM and the oxadiozolic oxygen ( $\mathrm{H} 4-\mathrm{O} 1$, black), oxadiozolic nitrogen ( $\mathrm{H} 4-\mathrm{N} 1$, blue), and hydrazonic nitrogen (H4-N2, red). 

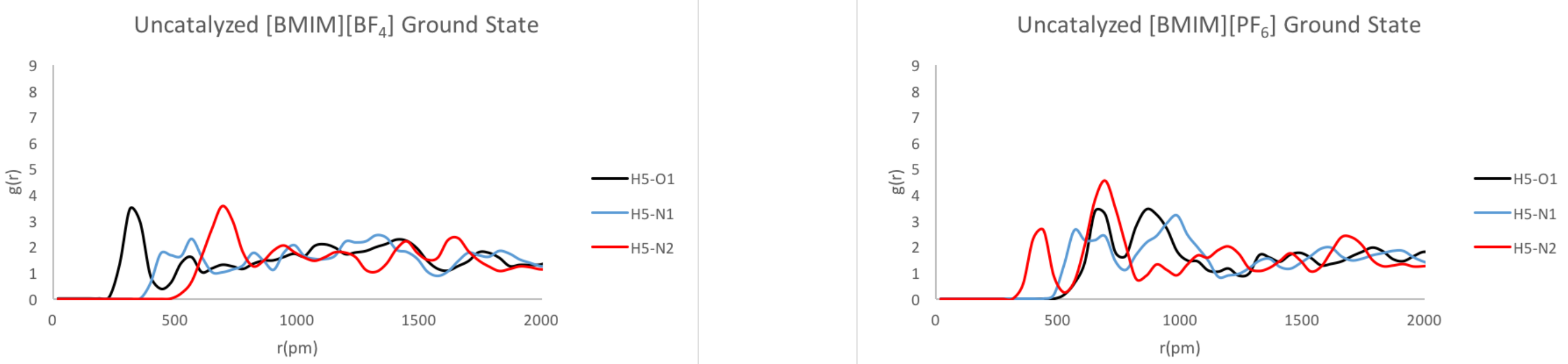

Uncatalyzed $[\mathrm{BMIM}]\left[\mathrm{BF}_{4}\right]$ Transition State

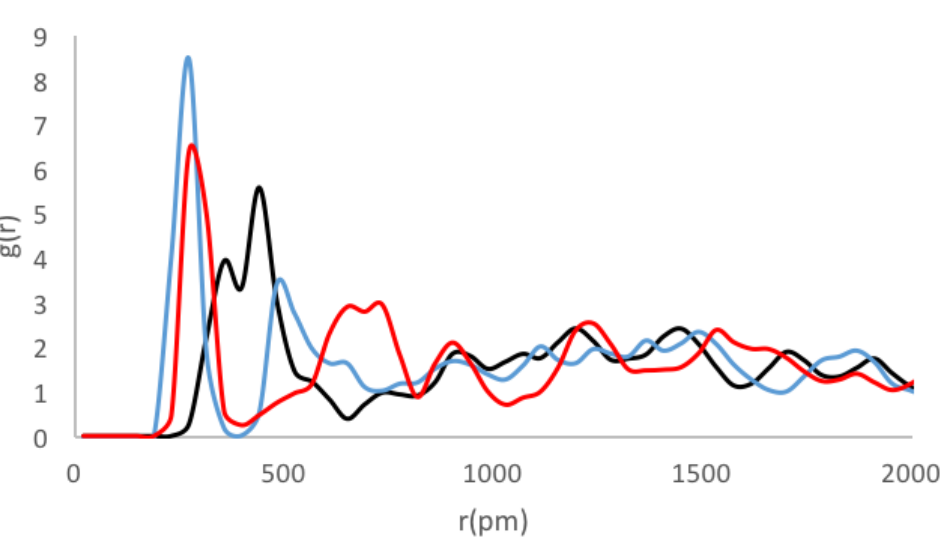

Uncatalyzed $[\mathrm{BMIM}]\left[\mathrm{PF}_{6}\right]$ Transition State

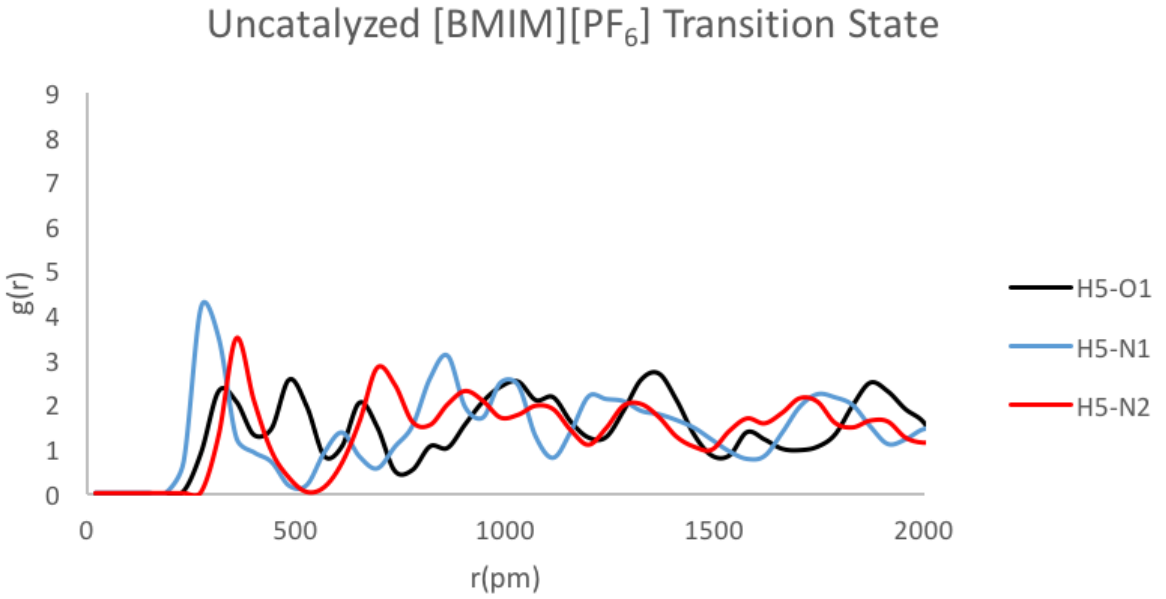

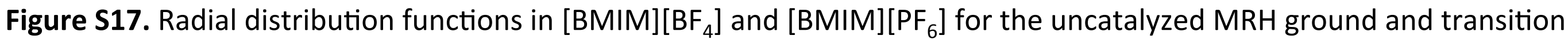
states between the $\mathrm{H} 5$ proton on BMIM and the oxadiozolic oxygen (H5-O1, black), oxadiozolic nitrogen (H5-N1, blue), and hydrazonic nitrogen (H5-N2, red). 


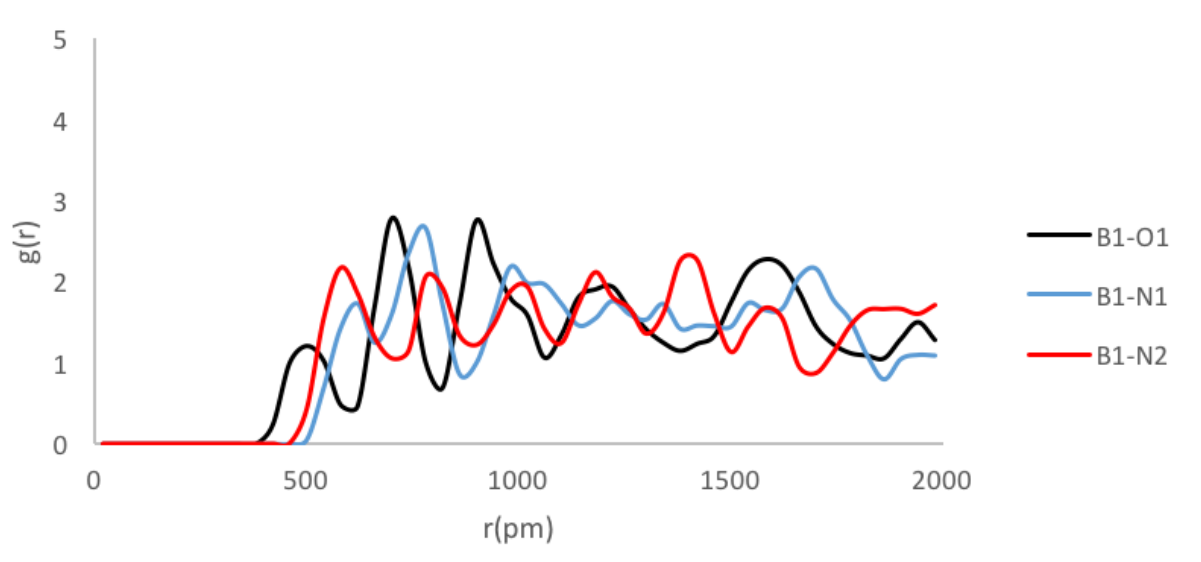

Specific Base Catalyzed [BMIM] $\left[\mathrm{BF}_{4}\right]$ Transition State

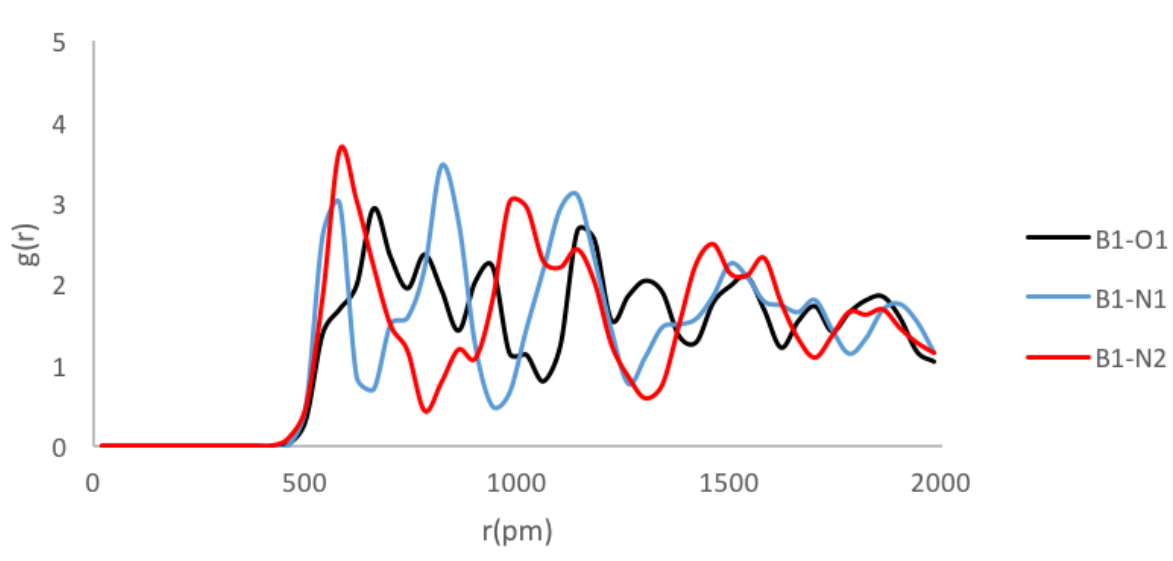

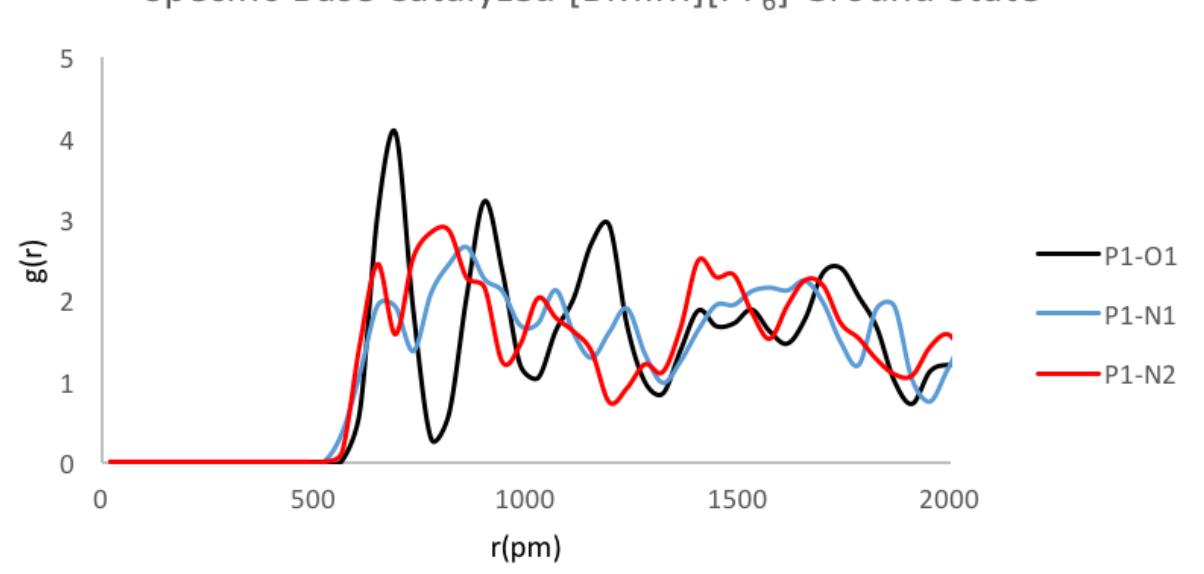

Specific Base Catalyzed [BMIM] $\left[\mathrm{PF}_{6}\right]$ Transition State

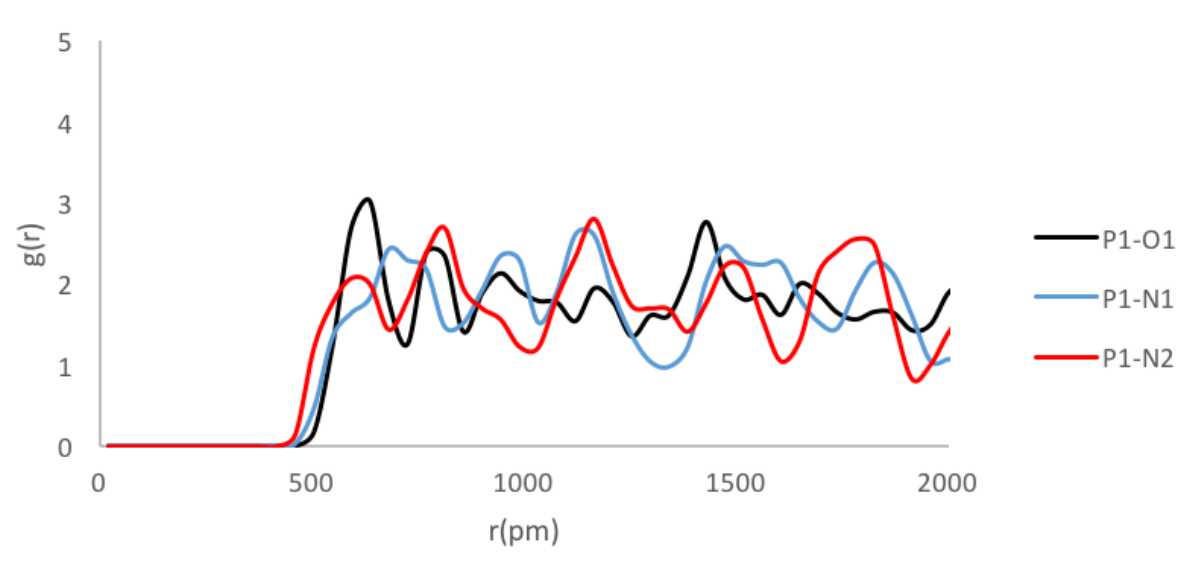

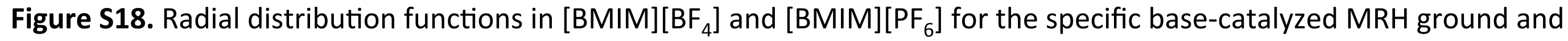
transition states between the center atom of the anion and the oxadiozolic oxygen (B1/P1-O1, black), oxadiozolic nitrogen (B1/ P1-N1, blue), and hydrazonic nitrogen (B1/P1-N2, red). 

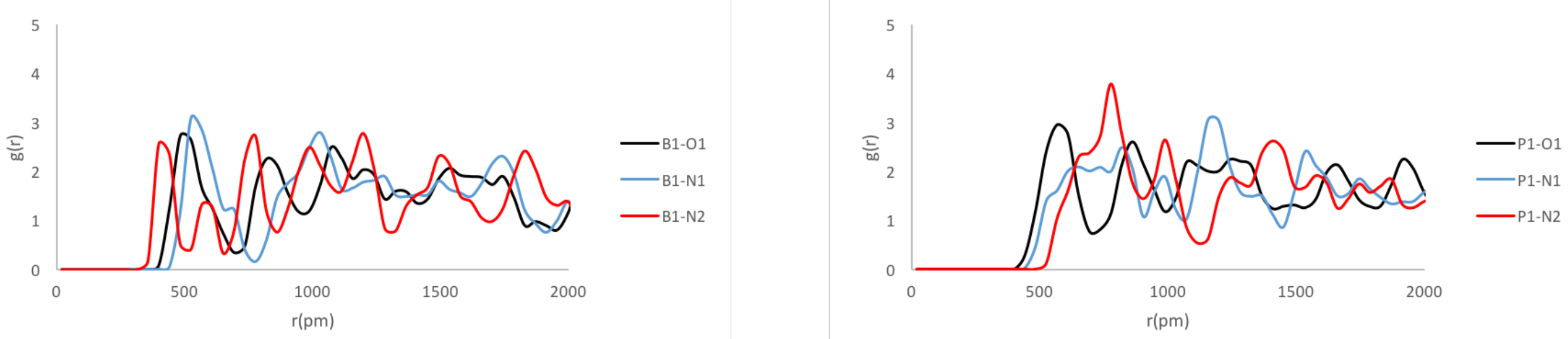

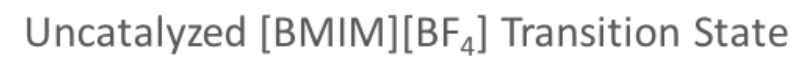

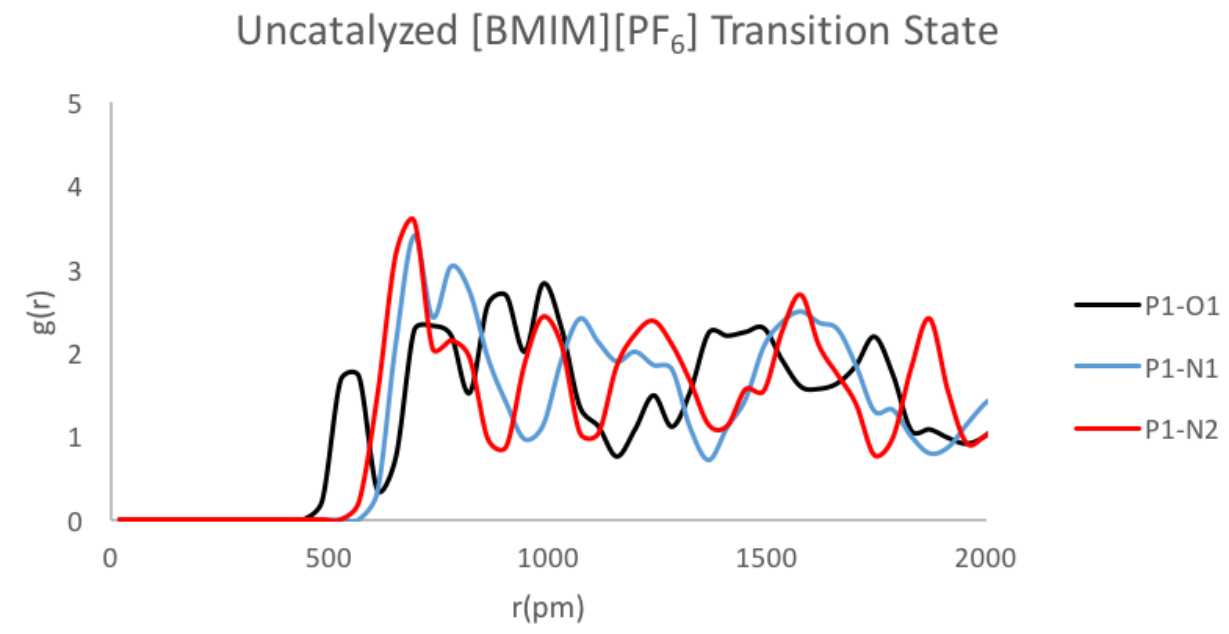

Figure S19. Radial distribution functions in $[B M I M]\left[B F_{4}\right]$ and $[B M I M]\left[\mathrm{PF}_{6}\right]$ for the uncatalyzed $M R H$ ground and transition states between the center atom of the anion and the oxadiozolic oxygen (B1/P1-O1, black), oxadiozolic nitrogen (B1/P1-N1, blue), and hydrazonic nitrogen (B1/P1-N2, red). 

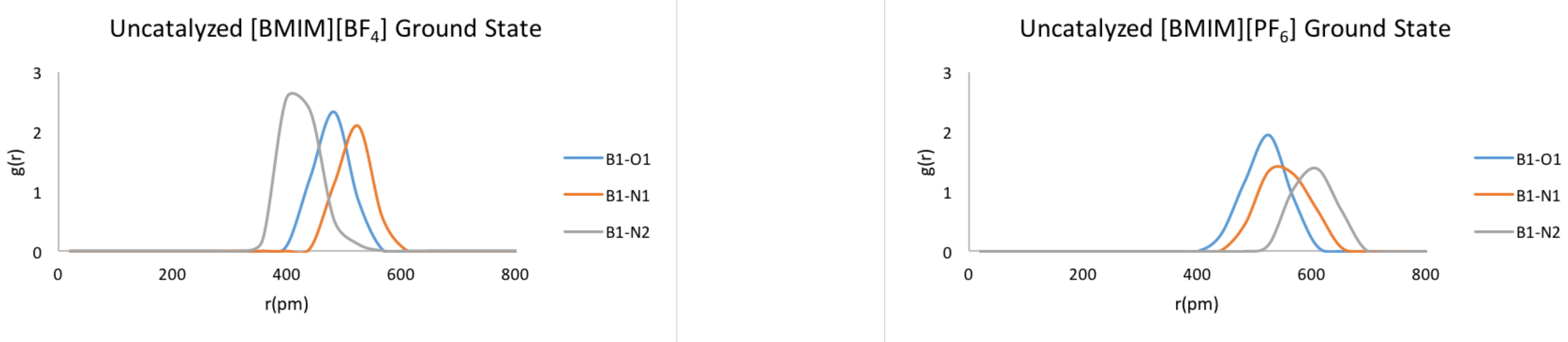

Uncatalyzed $[\mathrm{BMIM}]\left[\mathrm{BF}_{4}\right]$ Transition State

Uncatalyzed $[\mathrm{BMIM}]\left[\mathrm{PF}_{6}\right]$ Transition State
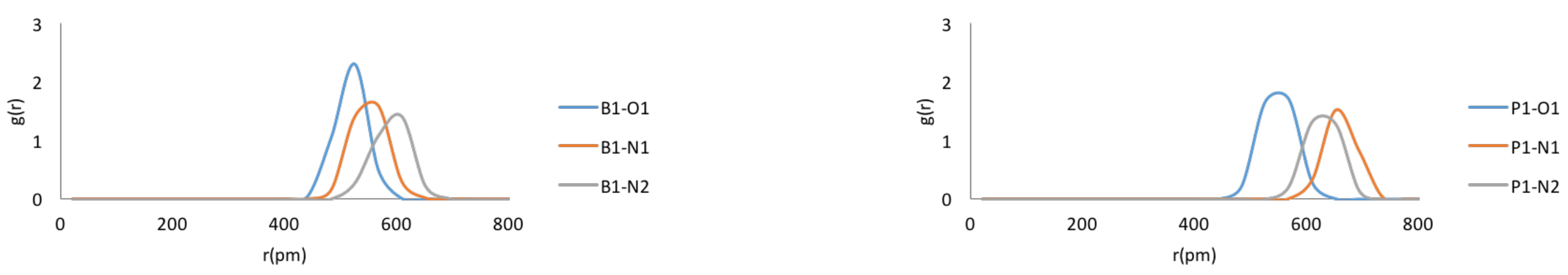

Figure S20. Nearest neighbor distribution between the center atoms of the nearest $\left[\mathrm{BF}_{4}\right]$ and $\left[\mathrm{PF}_{6}\right]$ anion and the oxadiozolic oxygen (B1/P1-O1, blue), oxadiozolic nitrogen (B1/P1-N1, orange), and hydrazonic nitrogen (B1/P1-N2, grey) in [BMIM] [BF 4 and $[\mathrm{BMIM}]\left[\mathrm{PF}_{6}\right]$ for the uncatalyzed mechanism. 
Specific Base Catalyzed [BMIM] $\left[\mathrm{BF}_{4}\right]$ Ground State

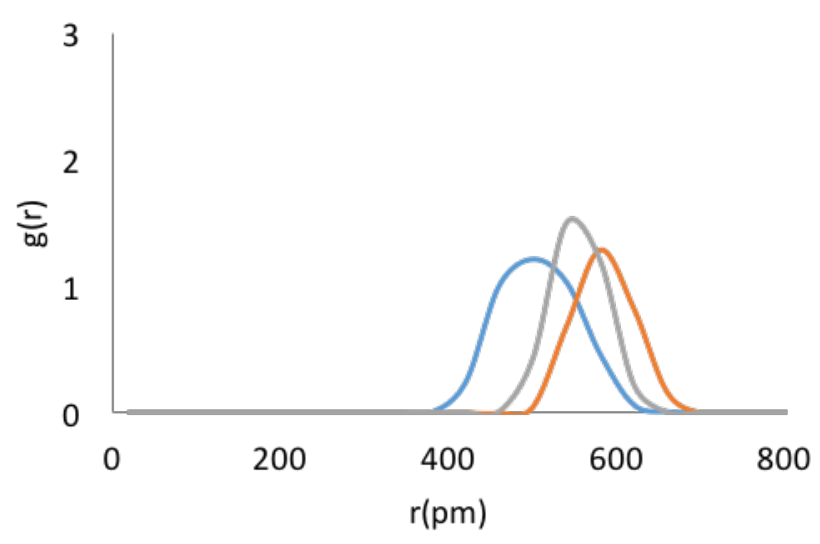

-B1-01

-B1-N1

-B1-N2

Specific Base Catalyzed [BMIM] $\left[\mathrm{BF}_{4}\right]$

Transition State

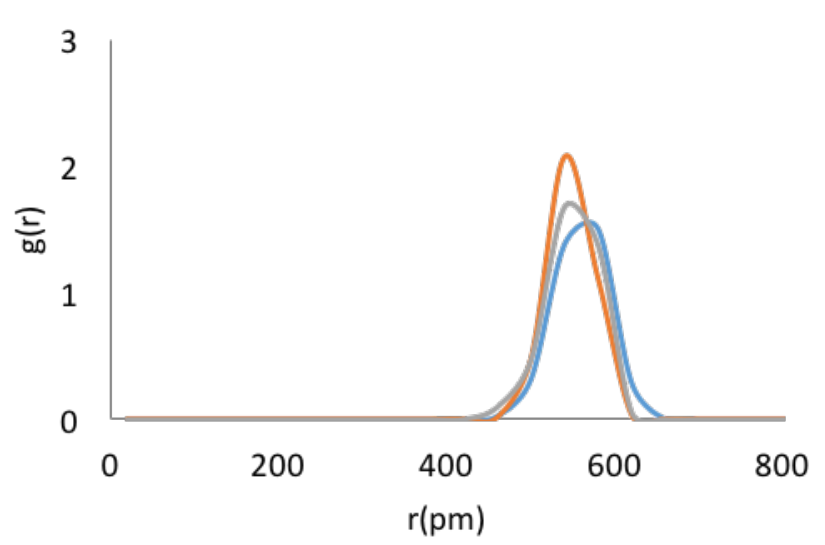

Specific Base Catalyzed [BMIM] $\left[\mathrm{PF}_{6}\right]$ Ground State

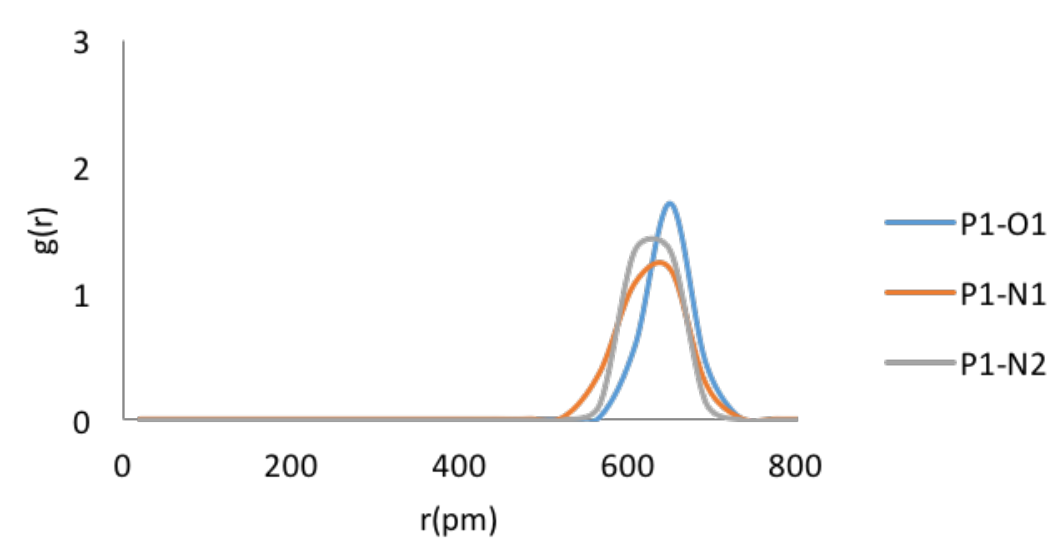

Specific Base Catalyzed [BMIM] $\left[\mathrm{PF}_{6}\right]$

Transition State

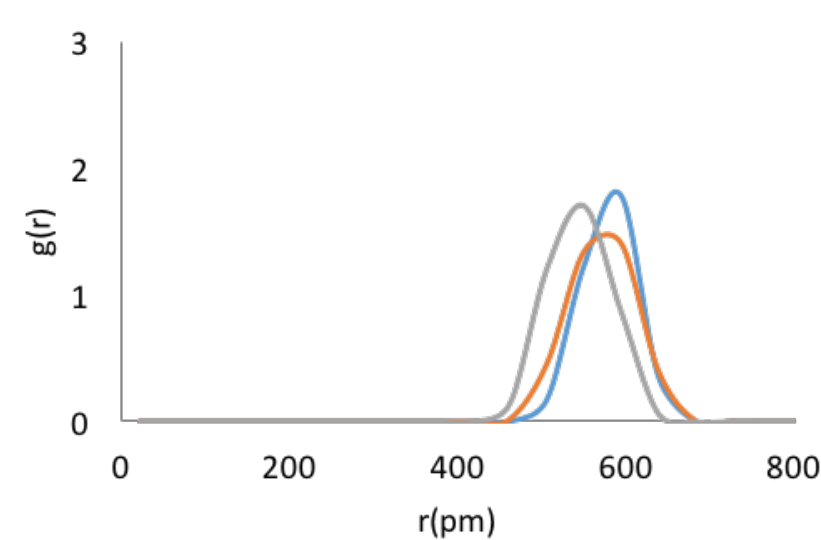

- P1-01

-P1-N1

B1-N1

$-\mathrm{B} 1-\mathrm{N} 2$

Figure S21. Nearest neighbor distribution between the center atoms of the nearest $\left[\mathrm{BF}_{4}\right]$ and $\left[\mathrm{PF}_{6}\right]$ anion and the oxadiozolic oxygen (B1/P1-O1, blue), oxadiozolic nitrogen (B1/P1-N1, orange), and hydrazonic nitrogen (B1/P1-N2, grey) in $[B M I M]\left[B F_{4}\right]$ and $[\mathrm{BMIM}]\left[\mathrm{PF}_{6}\right]$ for the specific base-catalyzed mechanism. 
Uncatalyzed $[\mathrm{BMIM}]\left[\mathrm{BF}_{4}\right]$ Ground State

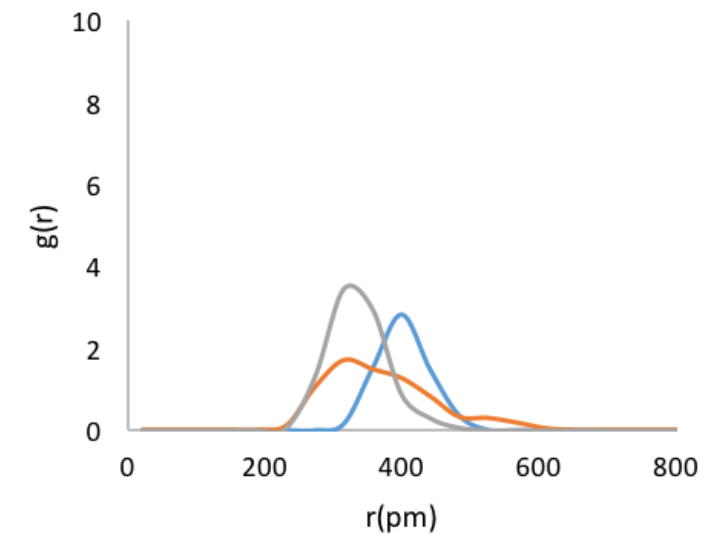

Uncatalyzed [BMIM] $\left[\mathrm{BF}_{4}\right]$ Transition State

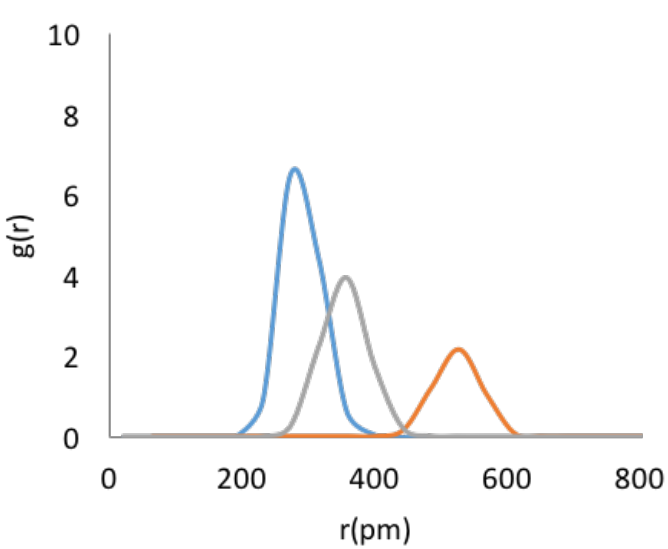

Uncatalyzed $[\mathrm{BMIM}]\left[\mathrm{BF}_{4}\right]$ Ground State

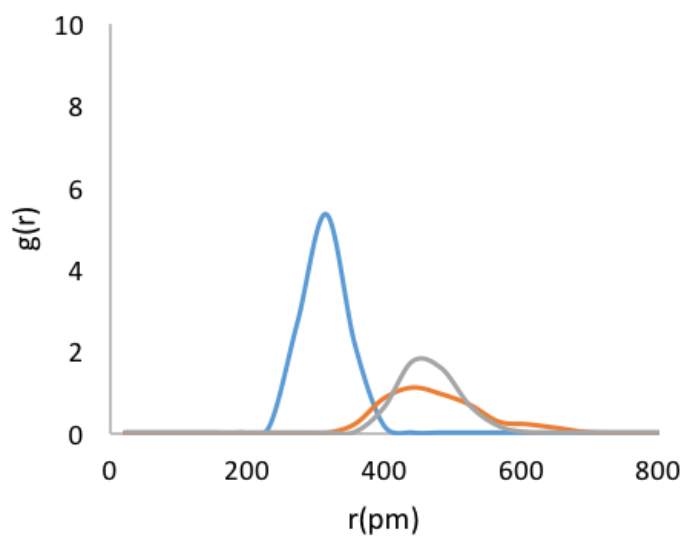

Uncatalyzed [BMIM] $\left[\mathrm{BF}_{4}\right]$ Transition State

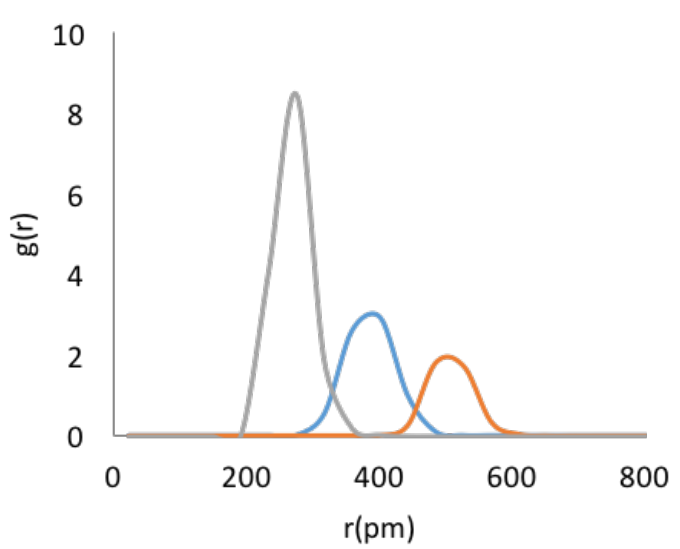

Uncatalyzed $[\mathrm{BMIM}]\left[\mathrm{BF}_{4}\right]$ Ground State

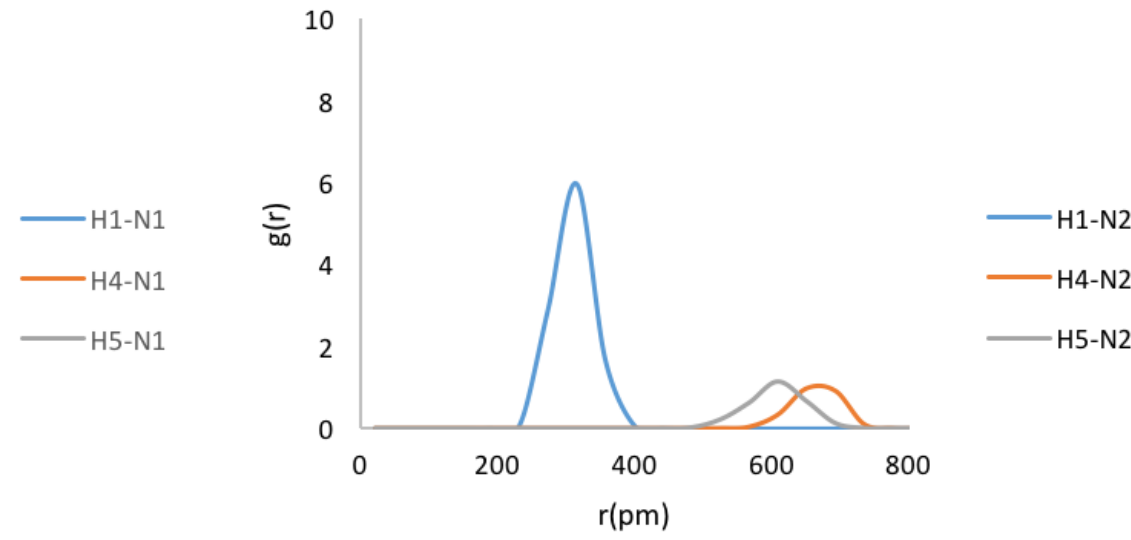

Uncatalyzed [BMIM] $\left[\mathrm{BF}_{4}\right]$ Transition State

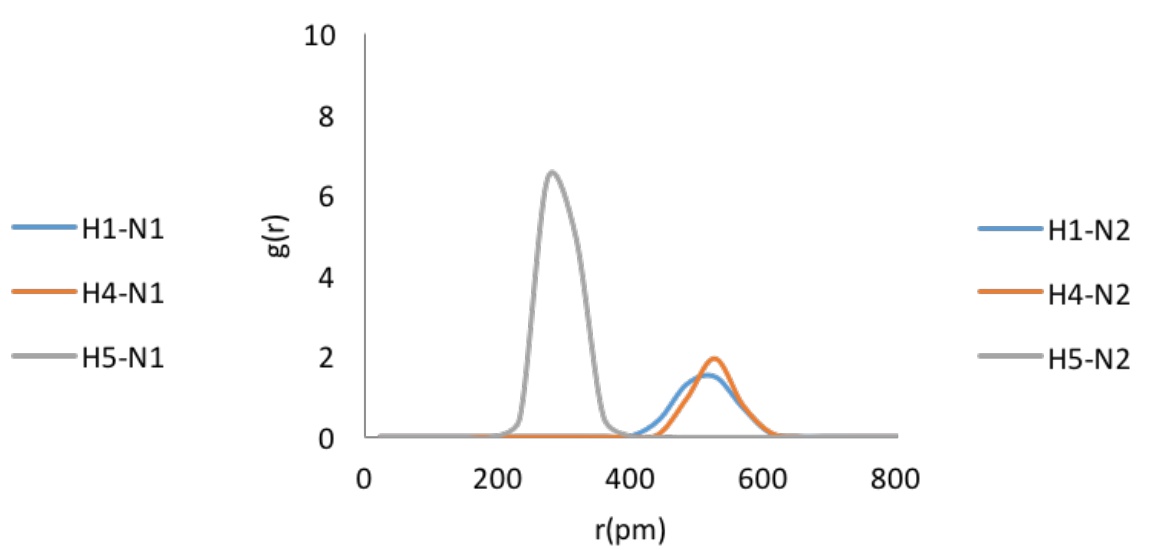

Figure S22. Nearest neighbor distribution between the H1 (blue), H4 (orange), and H5 (gray) protons on the imidazolium ring of [BMIM] and the oxadiozolic oxygen $(\mathrm{H}-\mathrm{O} 1)$, oxadiozolic nitrogen $(\mathrm{H}-\mathrm{N} 1)$, and hydrazonic nitrogen $(\mathrm{H}-\mathrm{N} 2)$ in $\left[\mathrm{BMIM}^{2}\left[\mathrm{BF}_{4}\right]\right.$ for the uncatalyzed mechanism. 
Specific Base Catalyzed [BMIM] $\left[\mathrm{BF}_{4}\right]$

Ground State

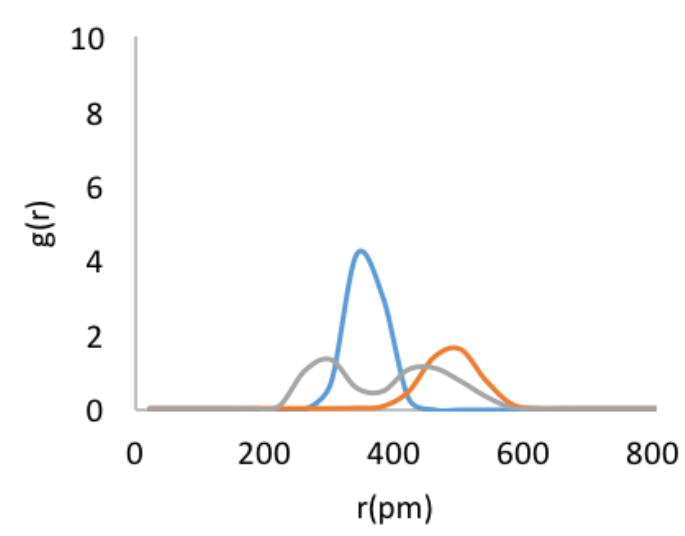

Specific Base Catalyzed [BMIM] $\left[\mathrm{BF}_{4}\right]$ Transition State

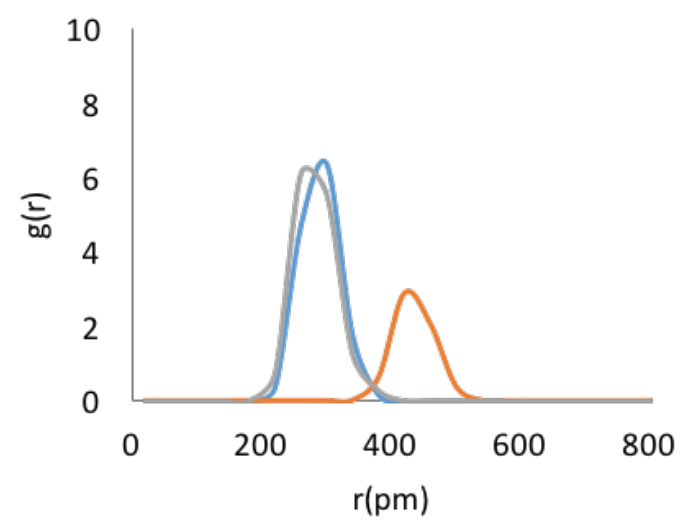

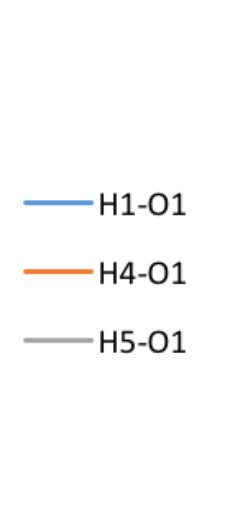

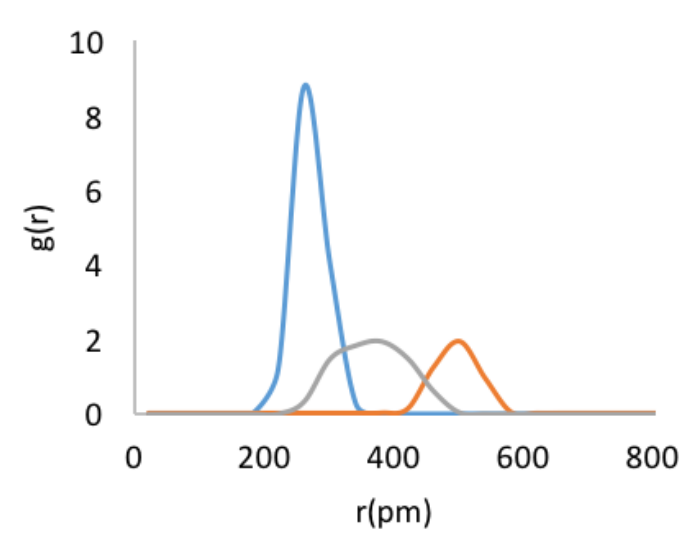

Specific Base Catalyzed [BMIM] $\left[\mathrm{BF}_{4}\right]$ Transition State
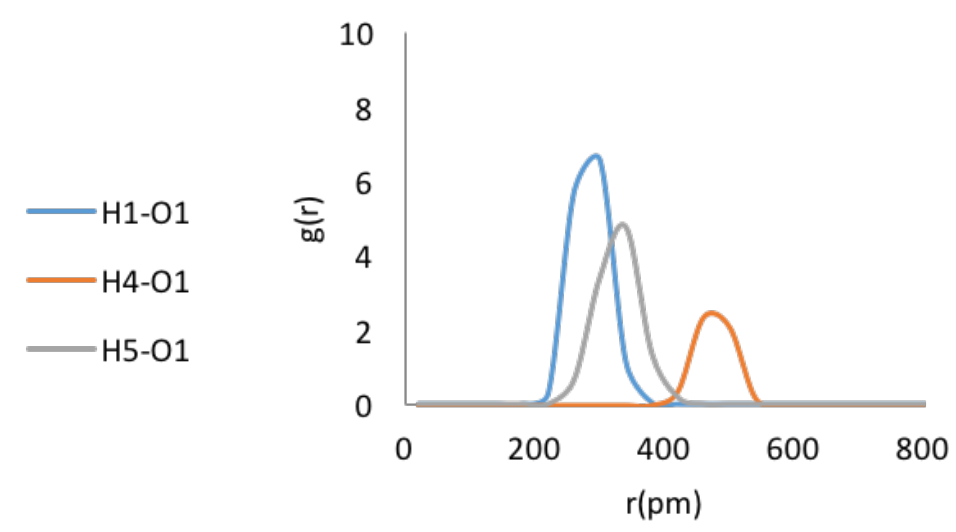

Specific Base Catalyzed [BMIM] $\left[\mathrm{BF}_{4}\right]$

Ground State

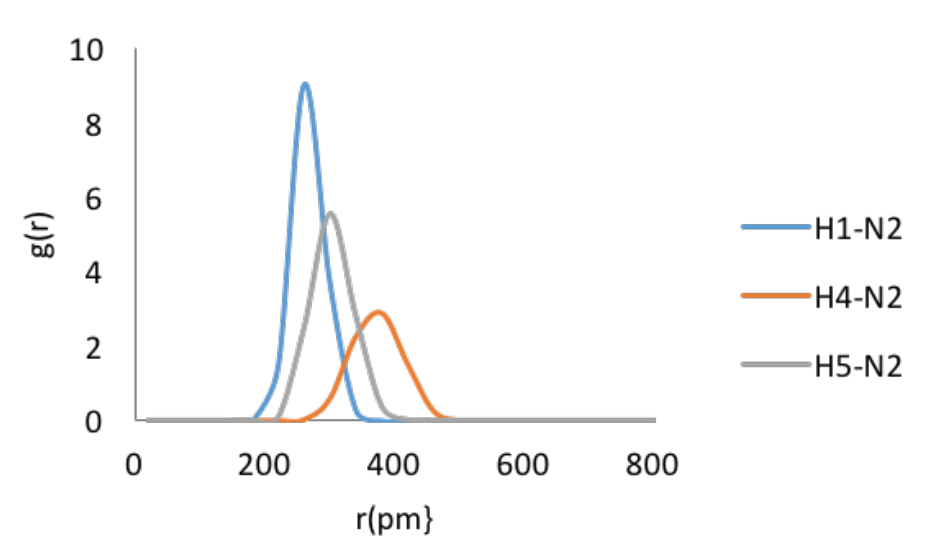

Specific Base Catalyzed [BMIM] $\left[\mathrm{BF}_{4}\right]$ Transition State
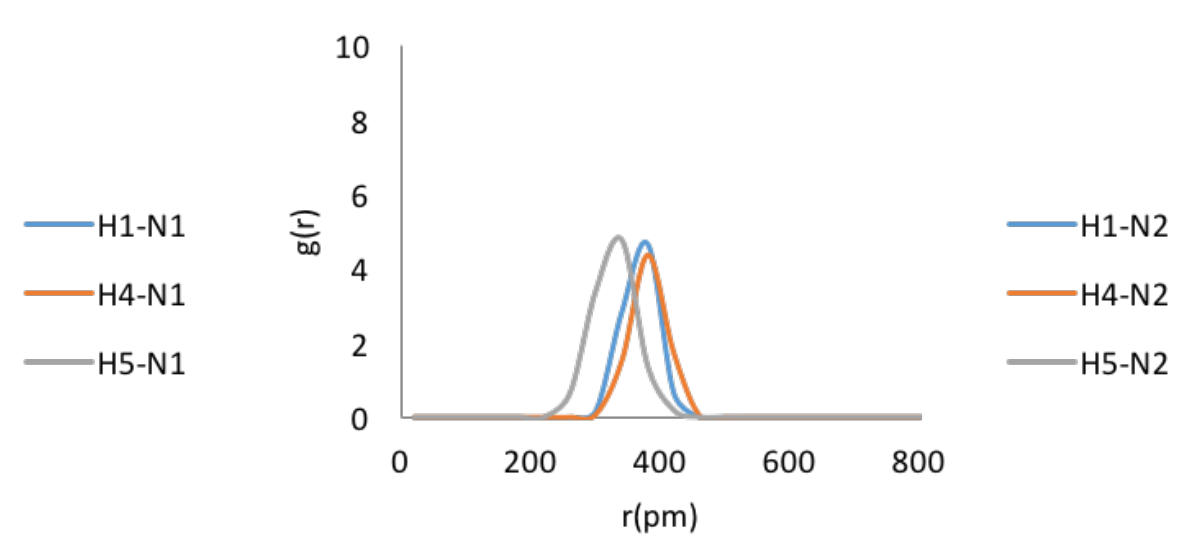

Figure S23. Nearest neighbor distribution between the $\mathrm{H} 1$ (blue), $\mathrm{H} 4$ (orange), and $\mathrm{H} 5$ (gray) protons on the imidazolium ring of [BMIM] and the oxadiozolic oxygen $(\mathrm{H}-\mathrm{O} 1)$, oxadiozolic nitrogen $(\mathrm{H}-\mathrm{N} 1)$, and hydrazonic nitrogen $(\mathrm{H}-\mathrm{N} 2)$ in $\left[\mathrm{BMIM}^{-}\left[\mathrm{BF}_{4}\right]\right.$ for the specific basecatalyzed mechanism. 
Uncatalyzed [BMIM] $\left[\mathrm{PF}_{6}\right]$ Ground State

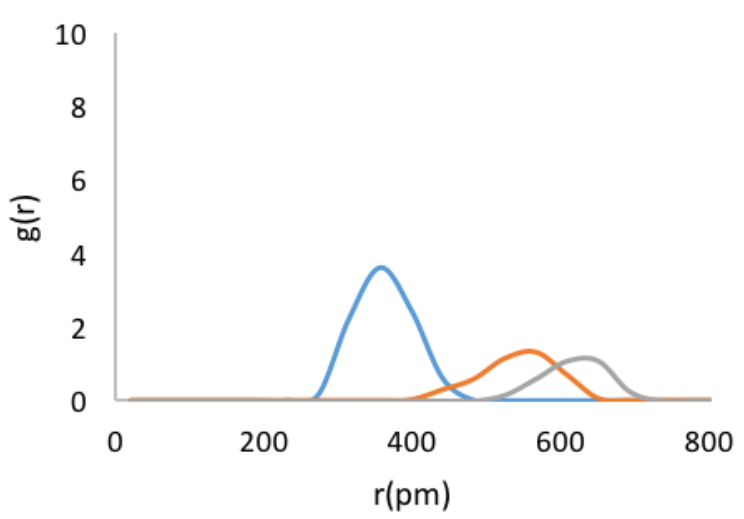

Uncatalyzed $[\mathrm{BMIM}]\left[\mathrm{PF}_{6}\right]$ Transition State

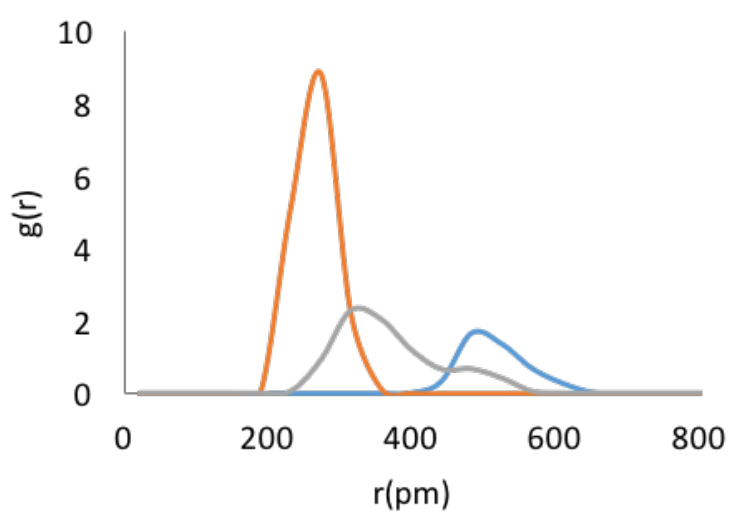

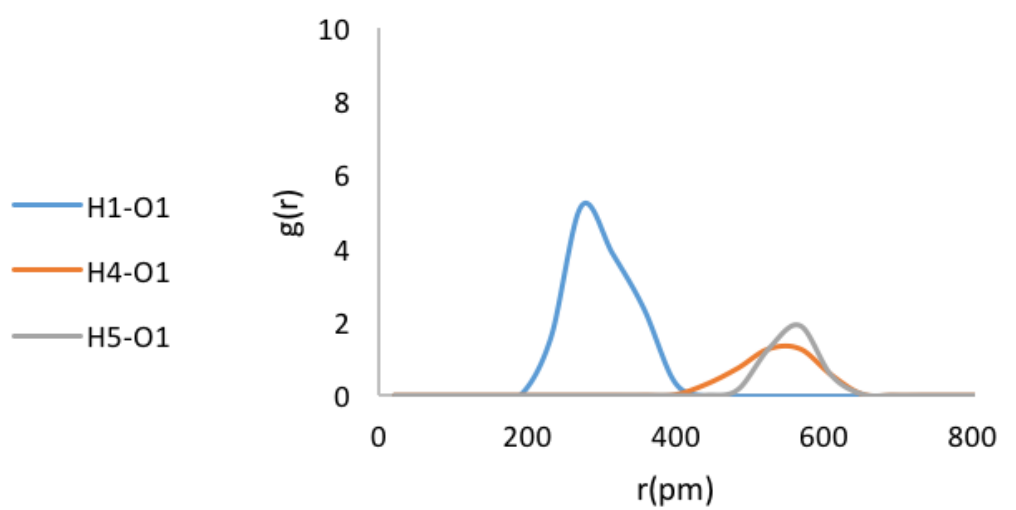

Uncatalyzed [BMIM] $\left[\mathrm{PF}_{6}\right]$ Transition State

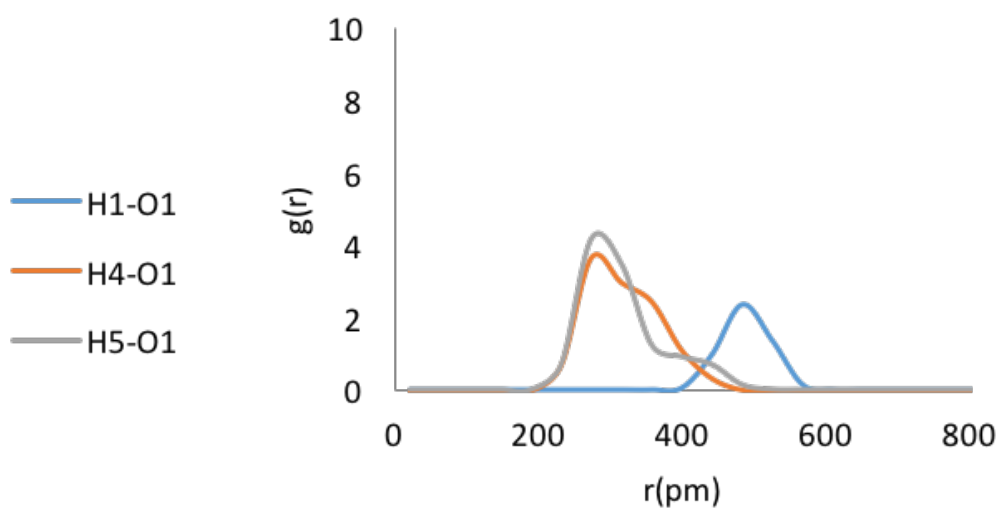

Uncatalyzed [BMIM] $\left[\mathrm{PF}_{6}\right]$ Ground State

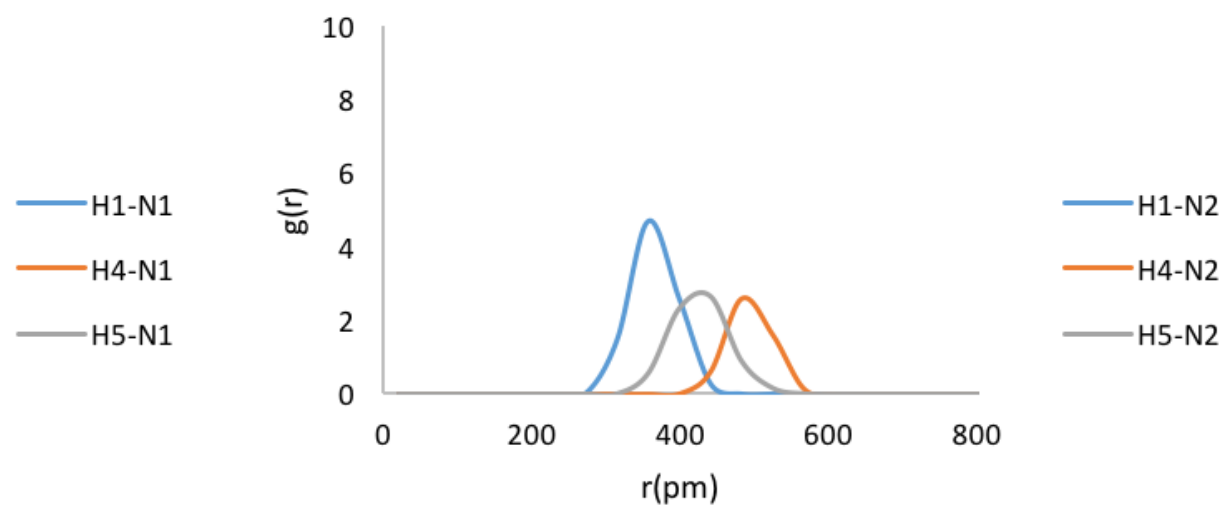

Uncatalyzed [BMIM] $\left[\mathrm{PF}_{6}\right]$ Transition State

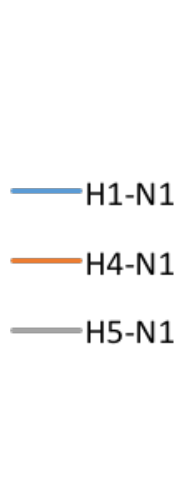

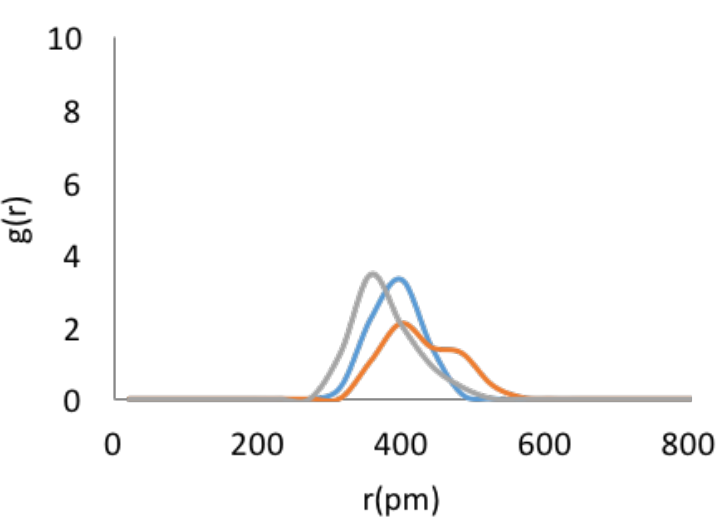

Figure S24. Nearest neighbor distribution between the $\mathrm{H} 1$ (blue), $\mathrm{H} 4$ (orange), and $\mathrm{H} 5$ (gray) protons on the imidazolium ring of [BMIM] and the oxadiozolic oxygen $(\mathrm{H}-\mathrm{O} 1)$, oxadiozolic nitrogen $(\mathrm{H}-\mathrm{N} 1)$, and hydrazonic nitrogen $(\mathrm{H}-\mathrm{N} 2)$ in $[B M I M]\left[\mathrm{PF}_{6}\right]$ for the uncatalyzed mechanism. 
Specific Base Catalyzed [BMIM] $\left[\mathrm{PF}_{6}\right]$

Ground State

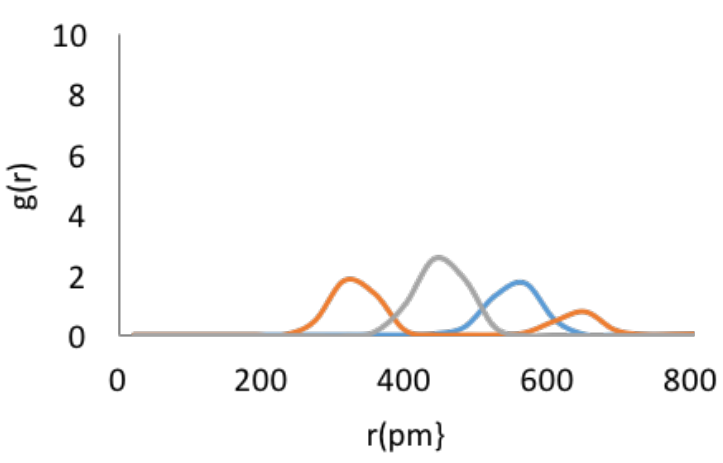

Specific Base Catalyzed [BMIM] $\left[\mathrm{PF}_{6}\right]$ Transition State

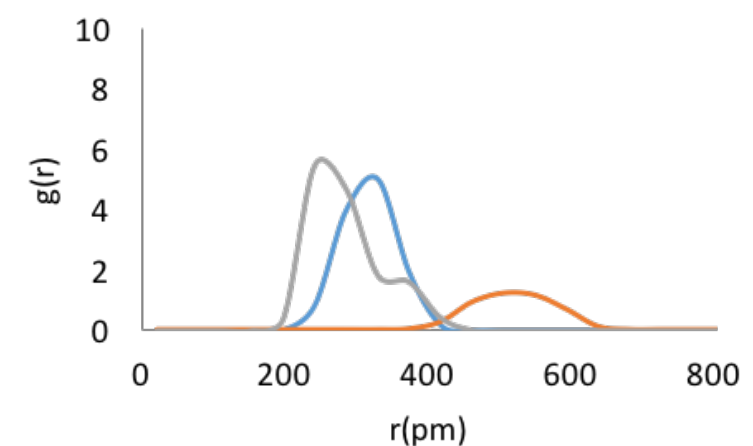

Specific Base Catalyzed [BMIM] $\left[\mathrm{PF}_{6}\right]$

Ground State
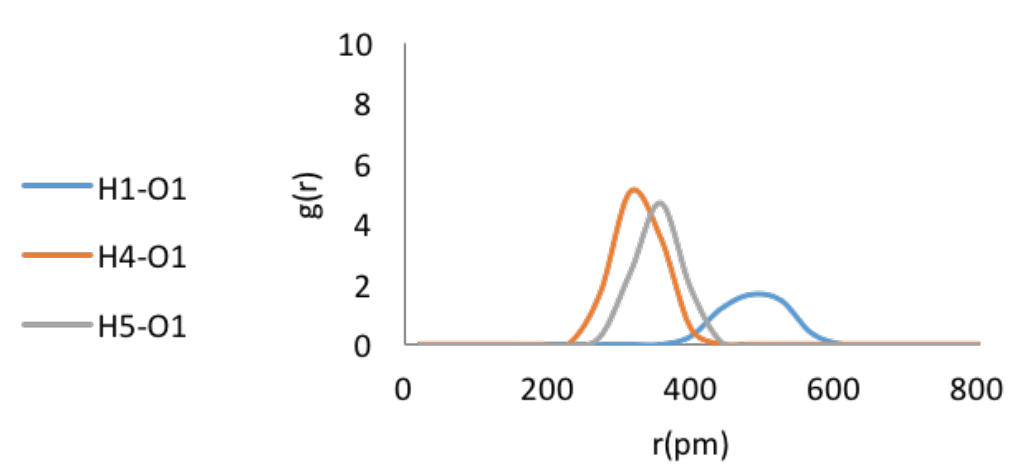

Specific Base Catalyzed [BMIM] $\left[\mathrm{PF}_{6}\right]$ Transition State
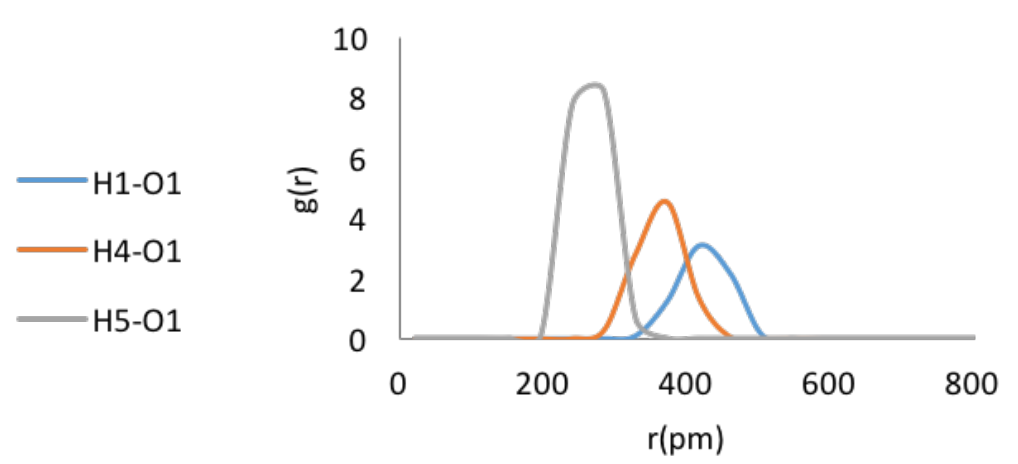

Specific Base Catalyzed [BMIM] $\left[\mathrm{PF}_{6}\right]$ Ground State
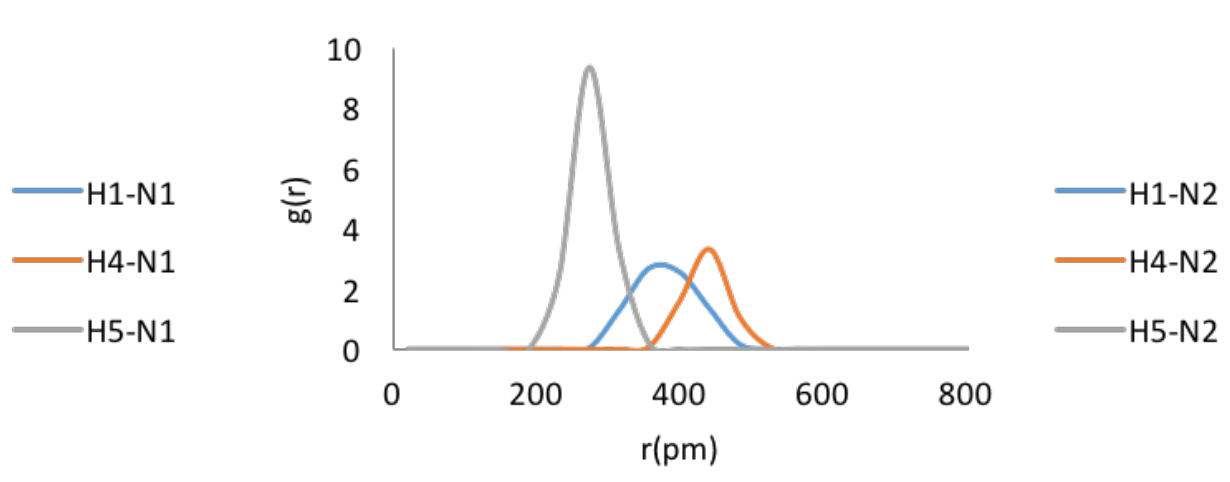

Specific Base Catalyzed [BMIM] $\left[\mathrm{PF}_{6}\right]$ Transition State

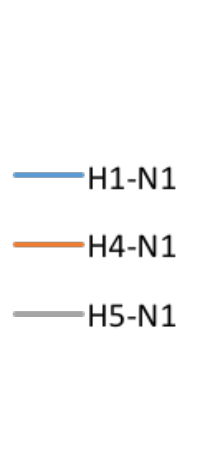

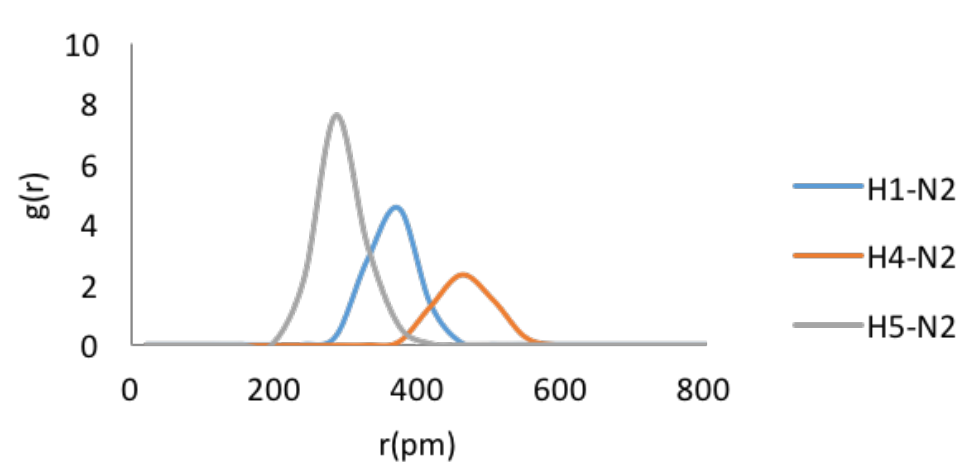

Figure S25. Nearest neighbor distribution between the H1 (blue), H4 (orange), and H5 (gray) protons on the imidazolium ring of [BMIM] and the oxadiozolic oxygen $(\mathrm{H}-\mathrm{O} 1)$, oxadiozolic nitrogen $(\mathrm{H}-\mathrm{N} 1)$, and hydrazonic nitrogen $(\mathrm{H}-\mathrm{N} 2)$ in $[\mathrm{BMIM}]\left[\mathrm{PF}{ }_{6}\right]$ for the specific base-catalyzed mechanism. 


\section{MRH transition structure in $\mathrm{CH}_{3} \mathrm{CN}$ using M06-2X/6-311+G(d,p)/CPCM}

\begin{tabular}{lrrr} 
& \multicolumn{1}{c}{1} & \multicolumn{1}{c}{2} & \multicolumn{1}{c}{3} \\
& \multicolumn{1}{c}{$\mathrm{A}$} & \multicolumn{1}{c}{$\mathrm{A}$} & $\mathrm{A}$ \\
Frequencies -- & -600.8027 & 22.4339 & 31.2220 \\
Red. masses -- & 13.7803 & 5.1774 & 3.9578 \\
Frc consts -- & 2.9307 & 0.0015 & 0.0023 \\
IR Inten -- & 1688.6808 & 3.8925 & 1.2894
\end{tabular}

Sum of electronic and zero-point Energies=

Sum of electronic and thermal Energies=

Sum of electronic and thermal Enthalpies=

Sum of electronic and thermal Free Energies=

$-1103.057548$

$-1103.037596$

$-1103.036652$

$-1103.109367$

$1 \backslash 1 \backslash$ ASN X86 64-DMC169 $\backslash$ Freq $\backslash$ RM062X \6-311+G (d,p) \C21H15N4O1 (1-) \AUBCRA \0

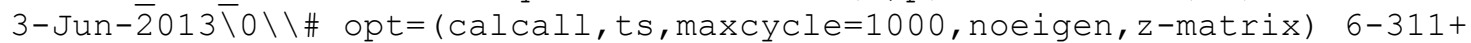
$\mathrm{g}(\mathrm{d}, \mathrm{p})$ scrf $=(\mathrm{cpcm}$, solvent=acetonitrile) $\mathrm{m} 062 \mathrm{x} \backslash \backslash \mathrm{Title}$ Card Required $\backslash \backslash-1$ $, 1 \backslash C, 5.6513128055,-2.4907858742,-0.1363979733 \backslash C, 4.2644984026,-2.402723$ $388,-0.0816340595 \backslash C, 4.4217545814,0.0079996771,-0.0312929761 \backslash C, 5.807157$ $5466,-0.0833176879,-0.0839631869 \backslash \mathrm{C}, 6.4239165679,-1.3321221339,-0.13750$ $55956 \backslash \mathrm{H}, 6.1288154719,-3.4624452857,-0.1790449585 \backslash \mathrm{H}, 3.9296053335,0.9721$ $195106,0.0110132926 \backslash \mathrm{H}, 6.4073948958,0.8188294255,-0.0838229259 \backslash \mathrm{H}, 7.5046$ $079339,-1.4015820273,-0.1796969834 \backslash C,-4.6425308816,-0.421642607,0.0170$ $979086 \backslash C,-5.8948526563,-1.0242532935,0.0115804953 \backslash C,-6.0251174157,-2.4$ $125704895,0.0164175601 \backslash \mathrm{C},-4.8731735574,-3.1995200632,0.0266655606 \backslash \mathrm{C},-3$ $.6164017382,-2.6104564162,0.0314435966 \backslash \mathrm{H},-4.5490005963,0.6566699839,0$. $0153594625 \backslash \mathrm{H},-6.7823827561,-0.4004763351,0.0045460852 \backslash \mathrm{H},-7.0059822177$, $-2.8720726676,0.0125187292 \backslash \mathrm{H},-4.9559771782,-4.2807580965,0.0304424966 \backslash$ $\mathrm{H},-2.7164212844,-3.214920513,0.0378540762 \backslash \mathrm{C}, 0.9709340562,4.2576618867$, $0.6133497975 \backslash C, 0.1266058184,5.1256659373,-0.0741537408 \backslash C,-0.9859656862$ $, 4.6077420665,-0.7334897354 \backslash C,-1.2438814092,3.2424311197,-0.7085014659$ $\backslash C,-0.4038146631,2.3560755983,-0.0155348482 \backslash C, 0.7112861912,2.890954980$ $7,0.6461571859 \backslash \mathrm{H}, 1.8371185057,4.6464953229,1.1372458309 \backslash \mathrm{H}, 0.3326264082$ $, 6.1894018244,-0.0966432571 \backslash \mathrm{H},-1.6512444394,5.2679470426,-1.2785910469$ $\backslash \mathrm{H},-2.1050021995,2.8445062588,-1.232121562 \backslash \mathrm{H}, 1.3726541655,2.2309985193$ $, 1.1902346293 \backslash \mathrm{C}, 3.6451327103,-1.1532186196,-0.030758442 \backslash \mathrm{C},-3.478762719$ $3,-1.2123115626,0.0260365935 \backslash \mathrm{N},-2.1829109439,-0.7058136639,0.032954383$ $6 \backslash \mathrm{N},-2.028884682,0.5754311204,0.000473852 \backslash \mathrm{C},-0.7566929302,0.9257174114$ $, 0.028923541 \backslash \mathrm{C}, 0.198320512,-0.1759153345,0.0586145464 \backslash \mathrm{C}, 2.1631290664,-$ $1.0626975033,0.023299505 \backslash \mathrm{N},-0.2181756925,-1.4408318096,0.0868506133 \backslash \mathrm{N}$, $1.5501325196,0.0989000729,0.0148538031 \backslash 0,1.4570438831,-2.1214400459,0$. $0738636877 \backslash \mathrm{H}, 3.6537860012,-3.2974578712,-0.0796189648 \backslash \backslash$ Version=EM64L-G 09 RevD.01 \State $=1-\mathrm{A} \backslash \mathrm{HF}=-1103.3709025 \backslash \mathrm{RMSD}=5.091 \mathrm{e}-09 \backslash \mathrm{RMSF}=4.021 \mathrm{e}-06 \backslash$ Zer oPoint $=0.3133548 \backslash$ Thermal $=0.3333068 \backslash$ Dipole $=2.2754985,1.1177169,-0.10901$ $33 \backslash$ DipoleDeriv $=-0.1919395,-0.1387471,-0.0068742,-0.1476699,0.0342527,0$ $.0109735,-0.0032911,0.0110444,-0.1771705,0.0145262,0.123904,-0.0056052$ $,-0.1146902,-0.0307807,0.0040329,-0.0012716,-0.003547,-0.1594697,-0.13$ $62828,-0.0787248,-0.0097362,0.1131423,-0.0443189,0.003628,-0.0040796,0$ $.0035114,-0.1421567,-0.0699089,0.1682137,0.0021757,0.1014676,0.0023816$ $, 0.0000367,0.000005,-0.0029585,-0.1834189,-0.035939,0.0202947,-0.00923$ $1,0.0508007,0.047759,0.0028886,-0.0025266,0.0036951,-0.1615581,0.03092$ $28,0.0756021,0.0060255,0.091023,-0.0530526,-0.0084536,0.0068356,-0.007$ $9262,0.1781778,0.1387913,0.0374747,0.0052438,0.0605485,0.0132319,-0.00$ $57866,0.0034917,-0.0042023,0.1787486,0.0077462,-0.074869,0.0032585,-0$. $0997483,-0.0280648,-0.0018296,0.0036565,-0.0029237,0.1776158,-0.095742$ $1,0.015287,0.0099201,0.0146686,0.1024873,-0.0024542,0.0085047,-0.00211$ 
$02,0.1771916,-0.9767946,-0.5095872,0.0013753,0.158246,0.0789704,0.0025$ $327,0.0005087,-0.0041095,-0.2265245,0.2118945,0.1991835,0.0002399,0.03$ $03755,0.2173196,-0.0029049,-0.0009014,-0.000316,-0.189957,-0.6443469,-$ $0.2072663,0.0015169,0.0237016,-0.3021167,0.0018542,0.0004767,-0.000325$ $2,-0.2386812,0.612542,0.1135372,0.0027305,0.0940726,0.1665417,-0.00176$ $54,0.0082903,0.0088624,-0.1914179,-0.5823133,-0.2530842,0.0003042,-0.9$ $92217,-0.5139983,-0.0011998,0.0083128,0.0011948,-0.2477039,0.1387401,0$ $.0136099,-0.0006154,0.0032068,-0.0411185,-0.0002873,-0.0044037,-0.0049$ $393,0.1969821,-0.1235841,0.0773815,-0.0015034,0.1160415,0.0368898,0.00$ $11213,-0.0023651,0.0001374,0.1751295,-0.1015849,-0.0867171,-0.0012968$, $-0.0867759,0.0480073,0.0001296,-0.0021101,-0.001041,0.2015113,0.083799$ $5,-0.018551,-0.000292,-0.0688111,-0.1403386,0.0012115,-0.0007981,0.000$ $7815,0.1768864,-0.0024433,0.0795296,-0.0005267,0.0869181,0.0457666,0.0$ $014274,-0.0026879,-0.0022107,0.1936957,-0.0120556,0.0130061,0.1272352$, $0.1055681,0.1924535,-0.0239554,0.1488082,0.0228846,-0.1050427,-0.14631$ $4,-0.0018254,0.0403272,-0.1034667,-0.3121058,-0.0094618,0.0589588,0.01$ $39654,-0.1829273,0.0014183,0.0439087,0.1164978,0.033244,0.1238776,-0.0$ $265636,0.1037779,-0.0748627,-0.1024487,0.0044162,0.2070795,0.0920048,-$ $0.1462662,-0.5092297,-0.0018319,0.0815156,0.0904772,-0.132196,0.071735$ $8,0.536789,0.0002598,0.5743979,1.5043568,-0.0328434,-0.018815,-0.08876$ $04,0.0267796,-0.1612814,-0.417197,0.0955192,-0.2128431,-0.4285338,-0.0$ $225469,0.0247669,-0.0996679,-0.1272004,-0.0260089,-0.0861857,-0.118755$ $,-0.0776131,0.0419842,-0.0260566,-0.1248052,-0.0520323,0.1036018,0.116$ $5892,-0.0436304,-0.0433162,-0.0519766,-0.1192828,0.0137181,-0.0419989$, $0.0150326,0.1605055,0.0495281,0.0824274,-0.0995636,0.0549799,-0.012776$ $3,0.0692683,-0.0959664,0.092134,0.0988058,0.0435053,-0.0542915,-0.1031$ $897,-0.0541095,0.0928645,-0.0297118,-0.095631,-0.0241248,0.110505,0.09$ $72995,0.054203,-0.0815103,0.0240684,0.1086271,0.0652092,-0.0890373,0.0$ $74857,0.1152422,-0.3571403,-0.0180709,0.0128539,-0.1434191,0.0805162,0$ $.0024424,0.0237375,-0.0039483,-0.0425226,2.9625036,1.6358335,0.0012943$ $, 1.9875909,0.6545254,0.0022609,-0.0058473,-0.0058781,0.1140366,-3.3484$ $553,-0.7664044,0.0074803,-3.602001,-2.4194199,-0.005546,-0.0400658,-0$. $0371609,-0.2190755,1.1523488,1.7598724,0.0043039,3.3115795,1.4919564,0$ $.0070682,0.1059248,0.2779692,-0.1952063,-2.4812511,-1.7710511,-0.02499$ $27,-0.1057891,-0.2457532,-0.004539,-0.0850154,-0.0779729,-0.1127711,2$. $4041096,-0.1511152,-0.0396467,-1.4859225,0.0978164,-0.0139167,-0.05503$ $49,-0.1258269,0.2211854,2.2777633,-0.0733297,-0.0488403,-0.2092221,1.2$ $608645,-0.0503774,-0.0726661,-0.0181692,0.3076349,3.4803285,-1.3236842$ $, 0.0144104,-0.8228675,0.0840879,0.0224291,0.0662658,0.0252072,-0.23621$ $88,-2.3653451,0.2975463,0.0139252,0.7444068,-0.931039,0.0150498,0.0730$ $406,-0.0382654,-0.3981518,-3.1697725,0.5934649,0.0354967,0.8385219,-1$. $4250194,0.0466377,0.026317,0.0421425,-0.3219121,0.1279948,-0.0738163,0$ $.0010961,-0.0931613,0.0294104,-0.0018886,-0.0038766,-0.0006171,0.17949$ $66 \backslash$ Polar $=652.8761605,65.2312405,512.017852,18.2362566,-5.9245451,220.9$ $579954 \backslash \mathrm{PG}=\mathrm{C} 01 \quad[\mathrm{X}(\mathrm{C} 21 \mathrm{H} 15 \mathrm{~N} 4 \mathrm{O} 1)] \backslash \mathrm{NImag}=1 \backslash \backslash$

\section{MRH ground state in $\mathrm{CH}_{3} \mathrm{CN}$ using M06-2X/6-311+G(d,p)/CPCM}

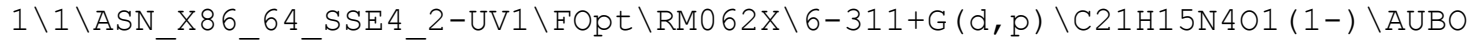
$\mathrm{XA} \backslash 02-\overline{\mathrm{M}} \mathrm{ay}-\overline{2} 01 \overline{4} \backslash 0 \backslash \backslash \overline{\#} \mathrm{m062x} / 6-311+\mathrm{g}(\mathrm{d}, \mathrm{p})$ opt $=(\mathrm{calcfc}, \operatorname{maxcycle}=1000$, noeig

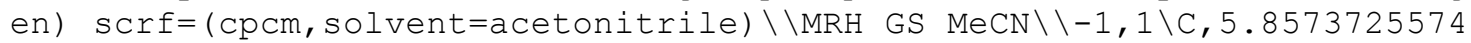
$,-2.3876706393,-0.0237114209 \backslash \mathrm{C}, 4.4810497364,-2.2783722499,0.1357415051$ $\backslash \mathrm{C}, 4.6142759523,0.0800914683,-0.3984727556 \backslash \mathrm{C}, 5.9887794599,-0.036436455$ $8,-0.5561252779 \backslash \mathrm{C}, 6.6116824766,-1.2691007371,-0.3688800931 \backslash \mathrm{H}, 6.3407295$ $445,-3.3459580138,0.1226358361 \backslash \mathrm{H}, 4.1168900266,1.0320283849,-0.53999813$ 
$92 \backslash \mathrm{H}, 6.5755550952,0.8336827581,-0.8246059002 \backslash \mathrm{H}, 7.6846315814,-1.3573000$ $171,-0.4920564118 \backslash \mathrm{C},-4.686242962,-0.2281052443,-0.1481412501 \backslash \mathrm{C},-6.0297$ $630573,-0.5650556645,-0.2618245389 \backslash \mathrm{C},-6.4475451688,-1.8952420968,-0.21$ $26736077 \backslash \mathrm{C},-5.4868789619,-2.8934320253,-0.04681787 \backslash \mathrm{C},-4.1424218194,-2$. $5666404257,0.0676479263 \backslash \mathrm{H},-4.3759203451,0.8079783952,-0.1888500127 \backslash \mathrm{H},-$ $6.7641359168,0.2233860672,-0.3918243007 \backslash \mathrm{H},-7.4972767503,-2.1474864112$, $-0.3023249729 \backslash \mathrm{H},-5.7889296193,-3.9346643846,-0.0066831958 \backslash \mathrm{H},-3.3962664$ $44,-3.3435563375,0.1964150692 \backslash \mathrm{C}, 0.6655234361,4.0928894178,0.81552047 \backslash \mathrm{C}$ $,-0.1798461283,4.8867857257,0.0436902597 \backslash \mathrm{C},-1.1932212926,4.2769708033$, $-0.6930609819 \backslash \mathrm{C},-1.3521683011,2.8961828478,-0.6630052155 \backslash \mathrm{C},-0.50856969$ $62,2.0812148119,0.1092308085 \backslash \mathrm{C}, 0.5025385662,2.7118915529,0.8505139162 \backslash$ $\mathrm{H}, 1.4524543853,4.5523080867,1.4035778656 \backslash \mathrm{H},-0.0507606949,5.9623970455$, $0.0170107606 \backslash \mathrm{H},-1.8573059702,4.8792403758,-1.3034880951 \backslash \mathrm{H},-2.137745935$ $9,2.4266441636,-1.2432477785 \backslash \mathrm{H}, 1.1610983669,2.1151381374,1.4694671517 \backslash$ $\mathrm{C}, 3.8585261845,-1.0426685487,-0.0529227001 \backslash \mathrm{C},-3.7069363833,-1.22893795$ $33,0.021454311 \backslash \mathrm{N},-2.3301821545,-1.0154244668,0.148284128 \backslash \mathrm{N},-2.00702096$ $38,0.2400441488,0.09038932 \backslash \mathrm{C},-0.7410769962,0.6188292322,0.1636124497 \backslash \mathrm{C}$ $, 0.398282261,-0.2851665719,0.246581662 \backslash \mathrm{C}, 2.4085839658,-0.9037356451,0$. $1131334067 \backslash \mathrm{N}, 0.3576738971,-1.5732181627,0.5334441941 \backslash \mathrm{N}, 1.6899593713,0$. $159733761,-0.0288454436 \backslash 0,1.6975892701,-1.9763543419,0.4491096933 \backslash \mathrm{H}, 3$. $8900284271,-3.1452827906,0.4052522283 \backslash \backslash V e r s i o n=E S 64 L-G 09 R e v D .01 \backslash$ State= $1-\mathrm{A} \backslash \mathrm{HF}=-1103.4005229 \backslash \mathrm{RMSD}=5.630 \mathrm{e}-09 \backslash \mathrm{RMSF}=3.993 e-06 \backslash \mathrm{Dipole}=4.4802182,0$. $8810462,-0.431216 \backslash$ Quadrupole $=1.8882273,0.6167707,-2.504998,-9.5233649$, $2.6290341,-0.2660753 \backslash \mathrm{PG}=\mathrm{CO} 1 \quad[\mathrm{X}(\mathrm{C} 21 \mathrm{H} 15 \mathrm{~N} 4 \mathrm{O} 1)] \backslash \backslash \mathrm{Q}$

\section{MRH transition structure in $\mathrm{CH}_{3} \mathrm{OH}$ using M06-2X/6-311+G(d,p)/CPCM}

\begin{tabular}{lrrr} 
& 1 & \multicolumn{1}{c}{2} & \multicolumn{1}{c}{3} \\
& \multicolumn{1}{c}{ A } & \multicolumn{1}{c}{ A } & \multicolumn{1}{c}{ A } \\
Frequencies -- & -600.6335 & 22.4667 & 31.0428 \\
Red. masses -- & 13.7799 & 5.1805 & 3.9489 \\
Frc consts -- & 2.9290 & 0.0015 & 0.0022 \\
IR Inten -- & 1685.1896 & 3.8824 & 1.3146
\end{tabular}

Sum of electronic and zero-point Energies=

Sum of electronic and thermal Energies=

Sum of electronic and thermal Enthalpies=

$-1103.057313$

$-1103.037362$

$-1103.036417$

Sum of electronic and thermal Free Energies=

$-1103.109131$

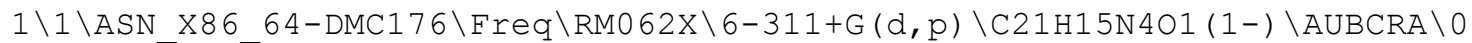

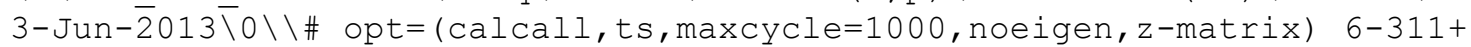
$\mathrm{g}(\mathrm{d}, \mathrm{p})$ scrf=(cpcm, solvent=methanol) $\mathrm{m062x \backslash \backslash Title}$ Card Required $\backslash \backslash-1,1 \backslash \mathrm{C}$ $, 5.6512641356,-2.4907672973,-0.1364429584 \backslash C, 4.2644478174,-2.4027213214$ $,-0.0816579353 \backslash C, 4.4217052569,0.0079954765,-0.0306792931 \backslash C, 5.807109384$ $7,-0.0833015897,-0.0833569305 \backslash C, 6.4238730247,-1.3320962065,-0.13723327$ $58 \backslash \mathrm{H}, 6.1287647165,-3.4624255839,-0.17935964 \backslash \mathrm{H}, 3.9295082445,0.972089685$ $6,0.0118475357 \backslash \mathrm{H}, 6.407345157,0.8188584494,-0.0829611495 \backslash \mathrm{H}, 7.5045674392$ $,-1.4015436225,-0.1794389572 \backslash C,-4.6425181476,-0.4216452651,0.016571419$ $2 \backslash C,-5.8948164282,-1.0242890037,0.0107423311 \backslash C,-6.0250490404,-2.412610$ $568,0.0151455848 \backslash C,-4.8730741426,-3.1995249206,0.0252741718 \backslash C,-3.61632$ $42903,-2.6104365546,0.0303572513 \backslash \mathrm{H},-4.5489789018,0.6566674153,0.015189$ $6558 \backslash \mathrm{H},-6.7823649355,-0.4005317741,0.003816551 \backslash \mathrm{H},-7.0059018143,-2.8721$ $408971,0.0110104402 \backslash \mathrm{H},-4.9558457096,-4.2807688299,0.0287181516 \backslash \mathrm{H},-2.71$ $62956761,-3.2148286798,0.0366798259 \backslash \mathrm{C}, 0.9709615627,4.2575437326,0.6140$ 
$451796 \backslash \mathrm{C}, 0.1266229482,5.125677418,-0.0732773259 \backslash \mathrm{C},-0.9859757235,4.6078$ $615221,-0.7326519049 \backslash \mathrm{C},-1.2439019856,3.2425557367,-0.7078770048 \backslash \mathrm{C},-0.4$ $038180307,2.3560644644,-0.0150964791 \backslash \mathrm{C}, 0.7113116054,2.8908354965,0.646$ $6359938 \backslash \mathrm{H}, 1.8371741519,4.6462806314,1.1379698418 \backslash \mathrm{H}, 0.3326546955,6.1894$ $16145,-0.095599034 \backslash \mathrm{H},-1.6512709976,5.2681554305,-1.2776273434 \backslash \mathrm{H},-2.105$ $0461296,2.8446992335,-1.2315053152 \backslash \mathrm{H}, 1.3726958963,2.2307735776,1.19055$ $84742 \backslash \mathrm{C}, 3.6450847609,-1.1532302178,-0.0304569344 \backslash \mathrm{C},-3.4787221156,-1.21$ $22825747,0.0253825416 \backslash N,-2.1829151815,-0.7057808762,0.0326156678 \backslash N,-2$. $0288978042,0.5754677799,0.0003280736 \backslash \mathrm{C},-0.7567091298,0.9257192452,0.02$ $91020532 \backslash \mathrm{C}, 0.1982959295,-0.1759387734,0.0588007467 \backslash \mathrm{C}, 2.1630653469,-1.0$ $627177333,0.0235409065 \backslash \mathrm{N},-0.2181844818,-1.4408520288,0.0867257108 \backslash \mathrm{N}, 1$. $5501267727,0.098892857,0.0153091203 \backslash 0,1.45699192,-2.1214662955,0.07378$ $27374 \backslash \mathrm{H}, 3.6536976296,-3.2974392141,-0.0799029774 \backslash \backslash$ Version=EM64L-G09ReV D. $01 \backslash$ State $=1-A \backslash H F=-1103.370669 \backslash \mathrm{RMSD}=3.961 \mathrm{e}-09 \backslash \mathrm{RMSF}=4.309 \mathrm{e}-06 \backslash$ ZeroPoint $=0.3133558 \backslash \mathrm{Thermal}=0.3333073 \backslash \mathrm{Dipole}=2.2742902,1.1155234,-0.1086916 \backslash \mathrm{Dip}$ oleDeriv $=-0.191487,-0.1385185,-0.0071427,-0.1473859,0.0343928,0.010950$ $1,-0.0034797,0.0110862,-0.177105,0.0140281,0.1237522,-0.0054027,-0.114$ $0391,-0.0306644,0.0041907,-0.0011926,-0.0035015,-0.1594153,-0.1368091$, $-0.0784358,-0.0095025,0.1127485,-0.0442232,0.0037393,-0.003906,0.00351$ $48,-0.1418079,-0.0694608,0.1679727,0.0020029,0.1011305,0.0025542,-0.00$ $00458,-0.000076,-0.0029159,-0.1834862,-0.0362196,0.020344,-0.0091832,0$ $.0507581,0.0474834,0.0031021,-0.0024662,0.003749,-0.161501,0.030776,0$. $0755798,0.0060995,0.0910853,-0.0531307,-0.0086012,0.0068636,-0.0079903$ $, 0.1780413,0.1389107,0.0374112,0.005294,0.0604129,0.0132542,-0.0058267$ $, 0.0034809,-0.0042241,0.1785022,0.0076538,-0.0748482,0.0033023,-0.0997$ $788,-0.0281848,-0.0018046,0.0036078,-0.0029847,0.1774695,-0.0959375,0$. $0153033,0.0098566,0.0146609,0.1023536,-0.0024984,0.0085101,-0.0021371$, $0.1770776,-0.9766692,-0.5096243,0.0012066,0.1582634,0.0789364,0.002868$ $7,0.0007416,-0.0039182,-0.226446,0.2123728,0.1991816,0.0005215,0.03081$ $67,0.2174539,-0.0028878,-0.00117,-0.0002775,-0.1899417,-0.6443391,-0.2$ $074759,0.001297,0.023474,-0.3019631,0.0015993,0.0006608,-0.0003328,-0$. $2385525,0.6127243,0.1139964,0.0027153,0.0939563,0.1666258,-0.0017167,0$ $.0081869,0.0089343,-0.1914119,-0.5820811,-0.2530424,0.0003808,-0.99198$ $72,-0.5141638,-0.0010575,0.0082539,0.0011924,-0.2472293,0.1386995,0.01$ $35726,-0.0005957,0.0032769,-0.041051,-0.0004621,-0.0044579,-0.0050361$, $0.1967503,-0.1236835,0.0774026,-0.001648,0.1160513,0.0367368,0.0011886$ $,-0.0023482,0.0001079,0.1749432,-0.1017625,-0.0867597,-0.0013779,-0.08$ $67935,0.0478739,0.0000533,-0.0021783,-0.001092,0.2013586,0.083677,-0.0$ $185425,-0.0002951,-0.0688563,-0.1405199,0.0012764,-0.0008378,0.0006814$ $, 0.1767459,-0.0022755,0.0794975,-0.0006208,0.0868095,0.0458359,0.00144$ $55,-0.0027143,-0.0022715,0.1935112,-0.011809,0.013057,0.1268658,0.1057$ $325,0.1928396,-0.0238395,0.1487311,0.0228129,-0.104822,-0.1464381,-0.0$ $019189,0.0407044,-0.10353,-0.3122913,-0.0097705,0.0589859,0.0139633,-0$ $.1830648,0.0017014,0.0441112,0.1160468,0.0332593,0.1242569,-0.0263865$, $0.1036659,-0.0747885,-0.1023202,0.0043448,0.2072305,0.0920553,-0.14642$ $03,-0.5094221,-0.0021946,0.081525,0.0905827,-0.1319536,0.0719066,0.536$ $8351,0.000411,0.5747343,1.5047363,-0.032293,-0.0186993,-0.0884047,0.02$ $68366,-0.1616163,-0.4175888,0.0955954,-0.2128515,-0.4286186,-0.0224142$ $, 0.0244918,-0.0998172,-0.1277172,-0.0261376,-0.0862339,-0.1186867,-0.0$ $775579,0.0418925,-0.0260144,-0.1247747,-0.0520541,0.1035195,0.1164538$, $-0.0436458,-0.0433012,-0.0519563,-0.1194792,0.0137559,-0.0419936,0.014$ $9937,0.1604011,0.0493801,0.082475,-0.0995526,0.0549844,-0.0129363,0.06$ $92593,-0.0959226,0.092133,0.0986974,0.0434229,-0.0541836,-0.1029879,-0$ $.0540368,0.0928429,-0.0296433,-0.0954414,-0.0241444,0.1104565,0.097177$ $1,0.0540514,-0.0815132,0.0239737,0.1086475,0.0651461,-0.0889561,0.0748$ $314,0.1151591,-0.3552229,-0.0182482,0.0125744,-0.1435928,0.0802085,0.0$ $022838,0.0238059,-0.0038795,-0.0424182,2.9610449,1.6354881,0.0023889,1$ 
$.9865102,0.6543809,0.002404,-0.0050666,-0.0055955,0.1137862,-3.3459932$ $,-0.7664629,0.006717,-3.5985858,-2.4181217,-0.0064332,-0.041276,-0.037$ $5273,-0.2194035,1.1509069,1.7586569,0.0048778,3.3083955,1.4913289,0.00$ $76498,0.1066526,0.2784258,-0.1944604,-2.478603,-1.7698882,-0.0260339,-$ $0.1069009,-0.246831,-0.0046531,-0.0854279,-0.078344,-0.1123103,2.40066$ $48,-0.1516266,-0.0391144,-1.4843511,0.0975939,-0.0142433,-0.0547252,-0$ $.1257344,0.2202161,2.2748815,-0.0735976,-0.0485677,-0.208562,1.2588537$ $,-0.0498647,-0.0727964,-0.0179474,0.3072716,3.4768118,-1.3223941,0.014$ $5971,-0.8221037,0.0842737,0.0224921,0.0665342,0.0251171,-0.2357357,-2$. $3624482,0.2976435,0.0137448,0.7431806,-0.9299222,0.0148279,0.0729294,-$ $0.0384501,-0.3974463,-3.1666325,0.5932887,0.0351482,0.8381809,-1.42328$ $05,0.0463573,0.0261754,0.0418972,-0.3215109,0.1280869,-0.0738156,0.001$ $1227,-0.0931061,0.0294474,-0.0019392,-0.0038959,-0.0006544,0.1793159 \backslash \mathrm{P}$ olar $=652.2809749,65.1911021,511.5305613,18.2678389,-5.847325,220.67846$ $36 \backslash \mathrm{PG}=\mathrm{CO} 1 \quad[\mathrm{X}(\mathrm{C} 21 \mathrm{H} 15 \mathrm{~N} 4 \mathrm{O} 1)] \backslash \mathrm{NImag}=1 \backslash \backslash$

\section{MRH ground state in $\mathrm{CH}_{3} \mathrm{OH}$ using M06-2X/6-311+G(d,p)/CPCM}

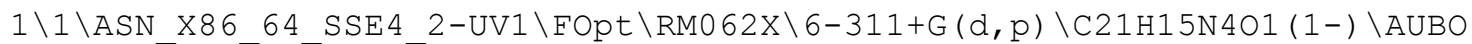
$\mathrm{XA} \backslash 02-\overline{\mathrm{M}} \mathrm{ay}-\overline{2} 01 \overline{4} \backslash 0 \backslash \backslash \overline{\#} \mathrm{m062x} / 6-311+\mathrm{g}(\mathrm{d}, \mathrm{p}) \quad$ opt $=(\mathrm{calcfc}, \operatorname{maxcycle}=1000$, noeig

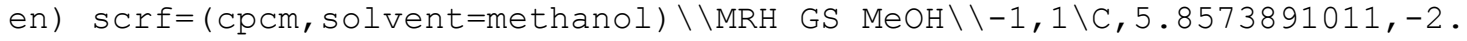
$3876647601,-0.0233908047 \backslash \mathrm{C}, 4.4810759574,-2.2783562984,0.1361654817 \backslash \mathrm{C}, 4$ $.6141626281,0.0799195598,-0.3988415576 \backslash \mathrm{C}, 5.9886537025,-0.0366136838,-0$ $.5565971784 \backslash \mathrm{C}, 6.6116332081,-1.2691866163,-0.3690002227 \backslash \mathrm{H}, 6.3407822781$, $-3.3458965349,0.1232196531 \backslash \mathrm{H}, 4.1167016894,1.0317781912,-0.5406323008 \backslash \mathrm{H}$ $, 6.5753708512,0.8334397449,-0.8254304145 \backslash \mathrm{H}, 7.6845733399,-1.3573875098$, $-0.4922607485 \backslash \mathrm{C},-4.6862109126,-0.2280572508,-0.147921998 \backslash \mathrm{C},-6.02971905$ $31,-0.5650302668,-0.2615656342 \backslash C,-6.4474349192,-1.8952574314,-0.212839$ $7429 \backslash \mathrm{C},-5.4866935418,-2.8934544275,-0.0474576842 \backslash \mathrm{C},-4.1422417799,-2.56$ $66464636,0.0669614413 \backslash \mathrm{H},-4.3759104802,0.8080508623,-0.1882221738 \backslash \mathrm{H},-6$. $764143873,0.2234325319,-0.391167947 \backslash \mathrm{H},-7.4971680869,-2.1475234212,-0.3$ $024401488 \backslash \mathrm{H},-5.7886882696,-3.9347181622,-0.0076500844 \backslash \mathrm{H},-3.3959918191$, $-3.3435271501,0.1953722934 \backslash \mathrm{C}, 0.6657522222,4.0929019762,0.8152539492 \backslash \mathrm{C}$, $-0.1799399542,4.8868574717,0.0438474947 \backslash \mathrm{C},-1.193609666,4.2770599818,-0$ $.692521111 \backslash \mathrm{C},-1.3525163411,2.8962762294,-0.6625178171 \backslash \mathrm{C},-0.5085799937$, $2.0812308582,0.1092883785 \backslash \mathrm{C}, 0.5028141304,2.711902089,0.8502049528 \backslash \mathrm{H}, 1$. $4529158966,4.552281654,1.4030364988 \backslash \mathrm{H},-0.0508922609,5.9624759235,0.017$ $2040613 \backslash \mathrm{H},-1.8579748568,4.8793544701,-1.3026211963 \backslash \mathrm{H},-2.1383396323,2.4$ $267520932,-1.2424353659 \backslash \mathrm{H}, 1.1616231812,2.1151320919,1.4688666719 \backslash \mathrm{C}, 3.8$ $584804046,-1.042746042,-0.0528388829 \backslash \mathrm{C},-3.7068324238,-1.2289059183,0.0$ $211824354 \backslash \mathrm{N},-2.3301098067,-1.0153694306,0.1480094992 \backslash \mathrm{N},-2.0069997321,0$ $.2400746257,0.0901968205 \backslash \mathrm{C},-0.7410545988,0.6188714895,0.1636302399 \backslash \mathrm{C}, 0$ $.3982763509,-0.2851407158,0.2468077841 \backslash \mathrm{C}, 2.4085420676,-0.9037781798,0$. $1133394627 \backslash \mathrm{N}, 0.3576812452,-1.5730987654,0.5340733399 \backslash \mathrm{N}, 1.6899604745,0$. $1596629468,-0.0288761762 \backslash 0,1.697591636,-1.9762873243,0.4497080894 \backslash \mathrm{H}, 3$. $8900806367,-3.1451804377,0.4060136422 \backslash \backslash V e r s i o n=E S 64 L-G 09 R e v D .01 \backslash$ State $=$ $1-\mathrm{A} \backslash \mathrm{HF}=-1103.400268 \backslash \mathrm{RMSD}=4.052 \mathrm{e}-09 \backslash \mathrm{RMSF}=3.899 \mathrm{e}-06 \backslash \mathrm{Dipole}=4.4767355,0.8$ $779771,-0.4304927 \backslash$ Quadrupole $=1.8404572,0.6217171,-2.4621742,-9.5221363$ $, 2.6216967,-0.2661078 \backslash \mathrm{PG}=\mathrm{C} 01 \quad[\mathrm{X}(\mathrm{C} 21 \mathrm{H} 15 \mathrm{~N} 4 \mathrm{O} 1)] \backslash \backslash \mathrm{C}$ 
Gaussian 09, Revision D.01,

M. J. Frisch, G. W. Trucks, H. B. Schlegel, G. E. Scuseria,

M. A. Robb, J. R. Cheeseman, G. Scalmani, V. Barone, B. Mennucci,

G. A. Petersson, H. Nakatsuji, M. Caricato, X. Li, H. P. Hratchian,

A. F. Izmaylov, J. Bloino, G. Zheng, J. L. Sonnenberg, M. Hada,

M. Ehara, K. Toyota, R. Fukuda, J. Hasegawa, M. Ishida, T. Nakajima,

Y. Honda, O. Kitao, H. Nakai, T. Vreven, J. A. Montgomery, Jr.,

J. E. Peralta, F. Ogliaro, M. Bearpark, J. J. Heyd, E. Brothers,

K. N. Kudin, V. N. Staroverov, T. Keith, R. Kobayashi, J. Normand,

K. Raghavachari, A. Rendell, J. C. Burant, S. S. Iyengar, J. Tomasi,

M. Cossi, N. Rega, J. M. Millam, M. Klene, J. E. Knox, J. B. Cross,

V. Bakken, C. Adamo, J. Jaramillo, R. Gomperts, R. E. Stratmann,

O. Yazyev, A. J. Austin, R. Cammi, C. Pomelli, J. W. Ochterski,

R. L. Martin, K. Morokuma, V. G. Zakrzewski, G. A. Voth,

P. Salvador, J. J. Dannenberg, S. Dapprich, A. D. Daniels,

O. Farkas, J. B. Foresman, J. V. Ortiz, J. Cioslowski,

and D. J. Fox, Gaussian, Inc., Wallingford CT, 2013. 